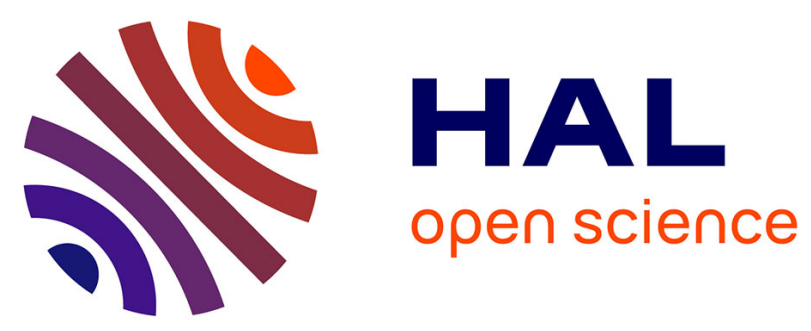

\title{
Vibrational levels of formaldehyde: Calculations from new high precision potential energy surfaces and comparison with experimental band origins
}

Andrei V Nikitin, Alexander E Protasevich, Alena A Rodina, Michael Rey, Attila Tajti, Vladimir G Tyuterev

\section{To cite this version:}

Andrei V Nikitin, Alexander E Protasevich, Alena A Rodina, Michael Rey, Attila Tajti, et al.. Vibrational levels of formaldehyde: Calculations from new high precision potential energy surfaces and comparison with experimental band origins. Journal of Quantitative Spectroscopy and Radiative Transfer, 2021, 260, pp.107478. 10.1016/j.jqsrt.2020.107478 . hal-03442408

\author{
HAL Id: hal-03442408 \\ https://hal.science/hal-03442408
}

Submitted on 23 Nov 2021

HAL is a multi-disciplinary open access archive for the deposit and dissemination of scientific research documents, whether they are published or not. The documents may come from teaching and research institutions in France or abroad, or from public or private research centers.
L'archive ouverte pluridisciplinaire HAL, est destinée au dépôt et à la diffusion de documents scientifiques de niveau recherche, publiés ou non, émanant des établissements d'enseignement et de recherche français ou étrangers, des laboratoires publics ou privés. 
Vibrational levels of formaldehyde:

calculations from new high precision potential energy surfaces and comparison with experimental band origins

Andrei V. Nikitin ${ }^{1}$, Alexander E. Protasevich ${ }^{1}$, Alena A. Rodina ${ }^{1}$, Michael Rey ${ }^{2}$, Attila Tajti ${ }^{3}$, and Vladimir G. Tyuterev ${ }^{2,4}$

${ }^{1 .}$ V.E. Zuev Institute of Atmospheric Optics, Russian Academy of Sciences, 1, Akademichesky Avenue, 634055 Tomsk, Russian Federation

2. Groupe de Spectrométrie Moléculaire et Atmosphérique, UMR CNRS 6089, Université de Reims, U.F.R. Sciences, B.P. 1039, 51687 Reims Cedex 2, France

3. ELTE Eötvös Loránd University, Institute of Chemistry, Laboratory of Theoretical Chemistry, P. O. Box 32, H-1518, Budapest 112, Hungary

${ }^{4}$ QUAMER, Tomsk State University, 36 Lenin Avenue, 634050 Tomsk, Russian Federation

Number of Pages: 27

Number of Figures: 8

Number of Tables: 9

Number supplemental files: 1

Running Head: Vibration energy levels of formaldehyde

Keywords: formaldehyde, potential energy surface, vibrational energy levels, kinetic energy operator

\section{Correspondence should be addressed to:}

Andrei V. Nikitin,

*Laboratory of Theoretical Spectroscopy, V.E. Zuev Institute of Atmospheric Optics, SB RAS, 1, Academician Zuev square, 634021, Tomsk, Russia

E-mail: avn@iao.ru

Phone : +73822-491111 (ext. 1208) 
Abstract

36

Vibrational energy levels of $\mathrm{H}_{2} \mathrm{CO}$ are reported using global variational nuclear motion calculations from new ab initio and empirically optimized full 6-dimensional ab initio potential energy surfaces in ground electronic state of the formaldehyde molecule. Ab initio calculations were carried out using extended electronic structure coupled-cluster calculations accounting for dynamic electron correlations including triple and quadruple excitations as well as relativistic and diagonal Born-Oppenheimer corrections. Variational nuclear motion calculations are compared in different set of coordinates with exact kinetic energy operator and in normal coordinates with Watson-Eckart kinetic energy operator. Our best ab initio potential energy surface including the above mentioned contributions provides the RMS (obs.-calc.) errors of 0.25 $\mathrm{cm}^{-1}$ for fifteen vibrational band centers without empirically adjusted parameters. The average error drops down to $0.08 \mathrm{~cm}^{-1}$ for an empirically optimized potential energy function with six adjusted parameters corresponding to quadratic force field terms. The estimation of the accuracy for the calculated vibrational levels in an extended range up to $4500 \mathrm{~cm}^{-1}$ shows that the set of ab initio vibrational levels could be more consistent than experimental levels obtained from earlier studies of low resolution spectra. The comparison of the calculated and experimental vibrational energy levels of the $\mathrm{D}_{2} \mathrm{CO}$ isotopologue is also reported.

\section{Introduction}

Accurate knowledge of spectroscopy of formaldehyde is important for the monitoring of this species in the atmosphere and also for detection of the presence of this molecule as an environmental pollutant indoors [1] [2] [3] [4]. Improving spectral parameters and line-by-line analyses are essential for reliable interpretation of near infrared spectra returned by the groundbased and orbiting observatories [5] [6]. An assignment of infrared spectra corresponding to high-energy overtone and combination bands is known to be a tedious and difficult task. Recent ab initio calculations of potential energy surfaces (PES) and of dipole moment surfaces (DMS) together with a progress in variational nuclear motion calculation have permitted a significant progress in predictions of vibration-rotation bands. Previous experience with some 4-5 atomic molecules [7] [8] [9] [10] [11] have shown that ab initio based approaches could be useful for better modelling and assignments of high-resolution spectra. In the present work, we report theoretical vibrational levels of formaldehyde computed from new accurate ab initio PES using the algorithms similar to one recently applied for the methane molecule [12] and compare our results with other recent calculations [13] [14].

From the theoretical standpoint, the formaldehyde molecule is an interesting case because of the rapid basis set convergence of variational calculations and a possibility to compare different 
methods conducted in various coordinate systems using high-level quantum-chemical calculations. Burleigh et al. [15] have been used the quadratic force field for formaldehyde obtained by refining the ab initio quartic force field CCST(T)/cc-pVTZ of Martin, Lee, and Taylor_[16] by means of iteratively fitting a subset of the coefficients of a Taylor-series expansion of the potential-energy surface to the set of the observed transition frequencies. Yachmenev et al. [14] have calculated ab initio PES using CCSD (T) / aug-cc-pVQZ method in a wide range of geometries. A detailed review of earlier works refs [17] [15] [16] [18] [19] [20] that reported calculations of the formaldehyde PES and energy levels can be found refs. [13] $[14]$.

The accuracy of ab initio calculations is in general lower than that of high-resolution experimental methods, except for studies of diatomic molecules with a small number of electrons. In the case of triatomics, very accurate ab initio calculations of potential energy surfaces (PES) and of dipole moment surfaces (DMS) led to a breakthrough in the extension of spectra analyses towards higher energy ranges. This was, for example, the case with the spectroscopy of water [21] [22] [23] [24], carbon dioxide [25] [26] [27], hydrogen sulfide [28] [29] and sulfur dioxide [30], as well as with the ozone spectra analyses [31] [32] [33]. In the latter case, ab initio predictions were mandatory for precise intensity quantification [34], for understanding the PES properties in the transition state range towards the dissociation threshold [35] [33], the interactions between potential wells [36], and modeling of the isotopic exchange reactions [37]. Many ab initio PESs have been subsequently refined by a fit to experimental data to achieve better accuracy in line positions. Theoretical line lists for ammonia [38] [27] and phosphine [39] [40] [41] are successful examples of this trend for four-atom species. New PESs and DMSs have been recently reported for five-atom [42] [43], six-atom molecules [44] [45], [46] and seven-atom [47] molecules.

Among the large number of ab initio calculations for many-electron systems, only two works reported calculations of the vibrational levels with the accuracy of about $0.1 \mathrm{~cm}^{-1}$, at least in the low and medium energy ranges: ref [24] [48] for the water molecule, and ref [12] for methane. While both of these molecules contain 10 electrons, the formaldehyde molecule has 16 electrons. In the recent study by Morgan et al. [13] the high accuracy vibrational fundamentals of formaldehyde have been reported, but not all calculated vibrational energy levels were published.

In contrast to methane, formaldehyde is an experimentally much less studied molecule. One of the possible reasons for this is the unavailability of the cold spectra caused by the high temperature of congelation and a complex technique for creating the formaldehyde gas. While 
for methane about 200 assigned vibrational energy levels have been measured with high accuracy, in case of formaldehyde, no more than 20 vibrational levels could be accurately obtained from the high resolution rotationally resolved spectra. The calculations reported in this paper can be considered as an additional source of information that could be useful for extended assignment and modeling of high-resolution experimental spectra of formaldehyde and its isotopologues.

This paper is structured as follows. The choice of the coordinates, the kinetic energy operator (KEO) and the analytical representations for the PES including their benchmark comparisons are discussed in Section 2. The dependence of the theoretical vibrational levels on the electronic basis set, the impact of relativistic corrections, diagonal Born-Oppenheimer corrections (DBOC), and of high-order electron correlation including connected triple and quadruple excitations in the coupled-cluster method is considered in Section 3. A detailed comparison with available experimental data in Sections 3 and 4 shows that the resulting fulldimensional ab initio PES provides currently the most accurate theoretical band origins for formaldehyde. We also discuss uncertainties and discrepancies in published experimental levels obtained from low- and high-resolution spectra. An empirically optimized PES obtained with a fine tuning of six PES parameters and the corresponding predicted vibrational levels are also reported.

\section{Coordinates and analytical PES representation}

For calculations of the vibrational-rotational energy levels of the formaldehyde molecule we used the kinetic energy operator (KEO) and PES written in terms of three sets of internal coordinates based on the angle-bisector molecule fixed axes [49], [50]. The sets of coordinates differ only in the definition of the internal vectors: (I) bond angle-bond length coordinates in which KEO was derived in ref [50], (II) Jacobi-like coordinates (see later), (III) Radau-Jacobi coordinates from refs [51] and [52]. The internal coordinates (I) and (II) are non-orthogonal sets while (III) is an orthogonal coordinate set. The kinetic energy operator of the nuclei (using the notation from ref [53]) in the laboratory frame

$$
\hat{T}_{L}=-\frac{1}{2} \hbar^{2} \sum_{k=1}^{4} \frac{1}{m_{k}} \frac{\partial^{2}}{\partial \vec{R}_{k}^{2}}
$$

can be written as a sum of the KEO of the center of mass and the $\underline{T}_{R_{-}}$relative motions of the nuclei, as follows: 


$$
\hat{T}_{R}=-\frac{1}{2} \hbar^{2} \sum_{i=1}^{3} \sum_{j=1}^{3} \frac{1}{m_{i, j}} \frac{\partial^{2}}{\partial \vec{r}_{i} \partial \vec{r}_{j}},
$$

where the effective masses are given by

$$
\frac{1}{m_{i, j}}=\sum_{k=1}^{4} \frac{A_{i k} A_{j k}}{m_{k}},
$$

where $m_{k}$ is the mass of the $\mathrm{k}^{\text {th }}$ nuclei, and the linear transformation of the 4 original nuclear position vectors $\vec{R}_{k}$ to new 3 internal vectors $\vec{r}_{i}$ is defined as follows:

$$
\vec{r}_{i}=\sum_{k=1}^{4} A_{i k} \vec{R}_{k}
$$

Let us use the following numbering of indices: 1- for the nucleus of the first hydrogen atom $\mathrm{H}_{1}, 2$ - for the nucleus of the second hydrogen atom $\mathrm{H}_{2}, 3$ - for oxygen $\mathrm{O}, 4$ - for carbon $\mathrm{C}$. The vectors $\vec{R}_{1}, \vec{R}_{2}, \vec{R}_{3}$, and $\vec{R}_{4}$ start from the same point and define the positions of the $\mathrm{H}_{1}, \mathrm{H}_{2}, \mathrm{O}$, and $\mathrm{C}$ nuclei, respectively. $m_{1}, m_{2}, m_{3}$, and $m_{4}$ are masses of the $\mathrm{H}_{1}, \mathrm{H}_{2}, \mathrm{O}$, and $\mathrm{C}$ nuclei, respectively. New vectors (1) $\vec{r}_{1}, \vec{r}_{2}$, and $\vec{r}_{3}$ define the relative positions of the $\mathrm{H}_{1}, \mathrm{H}_{2}$, and $\mathrm{O}$ nuclei with respect to different centers of mass. The differences in the coordinate systems are as follows: for (I), $\vec{r}_{1}, \vec{r}_{2}$, and $\vec{r}_{3}$ start from the C nucleus position; for (II), $\vec{r}_{1}, \vec{r}_{2}$, and $\vec{r}_{3}$ start from the $\mathrm{CH}_{1} \mathrm{H}_{2}$ center of mass; for (III), $\vec{r}_{1}$ and $\vec{r}_{2}$ start from the Radau canonical point for the $\mathrm{CH}_{1} \mathrm{H}_{2}$ group, and $\vec{r}_{3}$ starts from the $\mathrm{CH}_{1} \mathrm{H}_{2}$ center of mass. Figure 1 shows these three sets of the internal vectors. Table 1 gives the matrix elements $\left\|A_{i k}\right\|$ and values of $1 / m_{i, j}$ for these coordinate sets.

Table 1. Elements of the matrix of transformation to internal vectors and the effective masses for three sets of coordinates.

\begin{tabular}{|l|l|l|}
\hline $\begin{array}{l}\text { Set of } \\
\text { coordinates }\end{array}$ & $A_{i k}$ & $1 / m_{i, j}$ \\
\hline (I) & $\delta_{i k}-\delta_{4 k}$ & $\frac{1}{m_{i}} \delta_{i j}+\frac{1}{m_{4}}$ \\
\hline (II) & $\delta_{i k}+\frac{m_{k}}{m_{1}+m_{2}+m_{4}}\left(\delta_{3 k}-1\right)$ & $\frac{1}{m_{i}} \delta_{i j}+\frac{1}{m_{1}+m_{2}+m_{4}}\left(\delta_{3 i}+\delta_{3 j}-1\right)$ \\
\hline
\end{tabular}




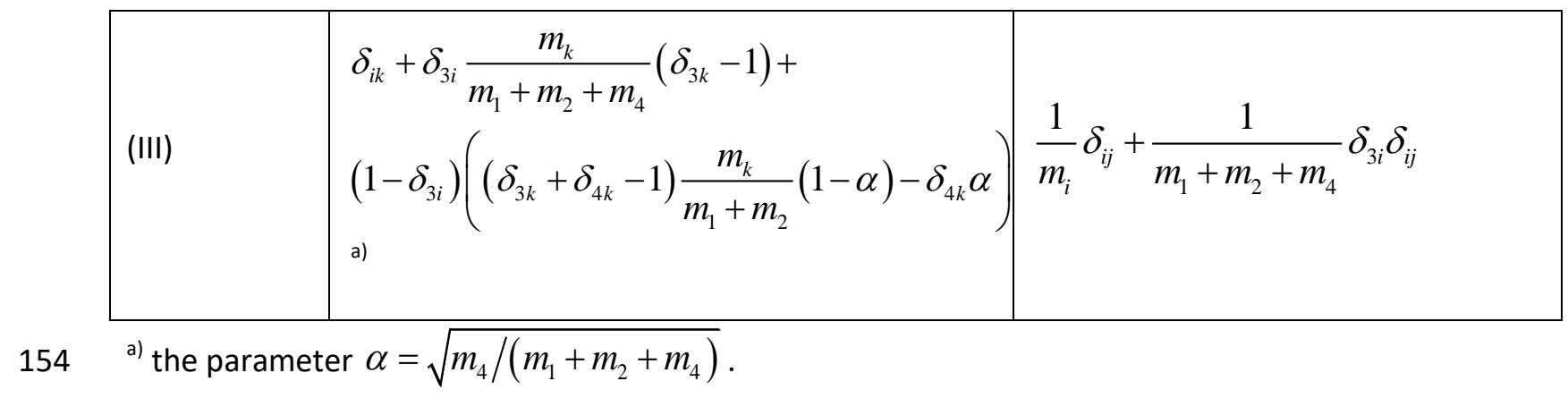

155

156
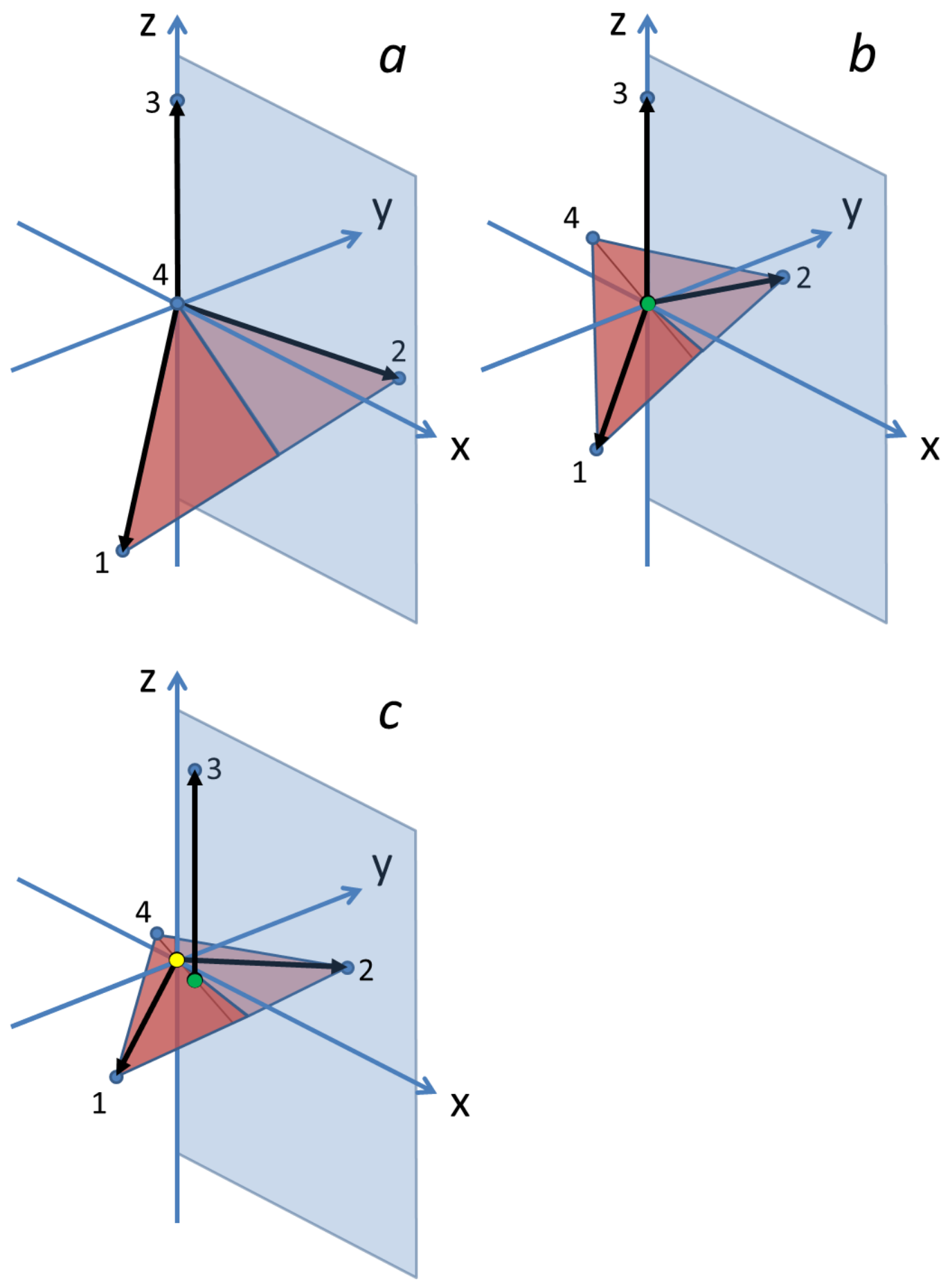
Figure 1. Internal vectors (depicted in black) for three sets of the internal coordinates based on the angle bisector coordinate system for the the formaldehyde molecule : a) bond angle-bond length coordinates (I), b) Jacobi-like coordinates (II), c) Radau-Jacobi coordinates (III). The 1, 2, 3, and 4 indices denote the $\mathrm{H}_{1}, \mathrm{H}_{2}, \mathrm{O}$, and $\mathrm{C}$ atom nuclei, respectively. Internal vectors with heads in the positions of nuclei 1,2 , and 3 have the following starting point: $a$ ) in the position of the nucleus $4, b$ ) in the center of mass of the nuclei 1,2 , and 4 (depicted in green) $c$ ) in the canonical point (depicted in yellow) of the nuclei 1, 2, and 4 (for vectors with heads in 1 and 2), and in the center of mass (depicted in green) of the nuclei 1,2 , and 4 (for the vector with head in 3 ).

Here we applied the same tensor techniques for an optimal sampling of the grid of nuclear configurations as described in the previous works for non-abelian symmetry groups [54] [55]. This permitted accounting for the full symmetry of the molecule in order to reduce the number of geometrical nuclear configurations for ab initio calculations of electronic energies. To build the corresponding grid of geometries, we used at the first step six one-dimensional sections(Figure 2 show three angular sections). The extent of the grid points was chosen in a way which ensures that a maximum number of parameters of our analytical PES representation would be well-defined in the least-squares fits to ab initio electronic energies. PES parameters responsible for the coupling of various vibrational modes were systematically included. The corresponding initial reference grid $\left(\mathrm{G}^{(\mathrm{R})}\right)$ contained 22509 points. This relatively dense grid provides a possibility for testing various high-order PES expansions in elementary functions of the symmetrized coordinates. The grid points were arranged in the order of increasing energy with the cut-off corresponding to the extent of the studied infrared bands. For this grid, the initial calculations were carried out with the coupled-cluster $\operatorname{CCSD}(\mathrm{T})$ method using the frozen core approximation and the quintuple-zeta cc-pV5Z basis set (that will be referred to as V5Z sets in abbreviated notation) and the MOLPRO program [56] [57].

The analytical PES was constructed using the irreducible tensor formulation described in refs [54] [55]. The PES can be expressed as a power series in elementary functions of the symmetrized coordinates involving Morse-type functions for "radial" and a sine function of angular variables:

$$
V\left(r_{1}, r_{C O}, r_{2}, \theta_{1}, \theta_{2}, \tau\right)=\sum_{i j k l m n} f_{i j k l m n} S_{1}^{i} S_{2}^{j} S_{3}^{k} S_{4}^{l} S_{5}^{m} S_{6}^{n} \text {, where }
$$

$S_{1}=\left(y\left(r_{1}\right)+y\left(r_{2}\right)\right) / \sqrt{2}, \quad S_{2}=y\left(r_{C O}\right), \quad S_{5}=\left(y\left(r_{1}\right)-y\left(r_{2}\right)\right) / \sqrt{2}$,

$S_{3}=\left(f\left(\theta_{1}\right)+f\left(\theta_{2}\right)\right) / \sqrt{2}, \quad S_{6}=\left(f\left(\theta_{1}\right)-f\left(\theta_{2}\right)\right) / \sqrt{2}, \quad f(\theta)=\sin \left(\theta-\theta_{e}\right)$.

A Morse-type function $y(r)=1-\exp \left(-a\left(r-r_{e}\right)\right)$ with the parameter $a=1.9$ is used as radial function. For the angles between bonds, the elementary function for the expansion is chosen as 

$f(\theta)=\sin \left(\theta-\theta_{e}\right)$. Note that the angular fitting that uses the function $f(\theta)=\sin \left((2 / 3)\left(\theta-\theta_{e}\right)\right)$

194

195

196

197

198

199

200

201

202

203

204

improves the accuracy of the fit to a minor extent. However, we did not use this function because it requires the numerical evaluation of one-dimensional integrals. The torsion angle $\tau$ is the angle between two planes $\vec{r}_{1} \times \vec{r}_{3}$ and $\vec{r}_{2} \times \vec{r}_{3}$. The following functions: $\cos (\tau)+1, \cos (\tau / 2), \tau-\pi$ have been tested as the torsion function $S_{4}(\tau)$. The first function transforms as the $\mathrm{A}_{1}$ irreducible representation while the second and third functions transform as $\mathrm{B}_{1}$. Contrary to the case of methane [12] [55], the expansion (2) does not contain components of degenerate vibrations due to the simplicity of the Abelian $\mathrm{C}_{2 \mathrm{v}}$ point group.

To validate various representations of the PES using the least-squares technique, an extended grid of geometries was used. This grid was augmented by adding supplementary sets of points corresponding to $180-\tau$ values with respect to all values of $\tau$ (for $\tau \neq 180$ ) in the initial grid.

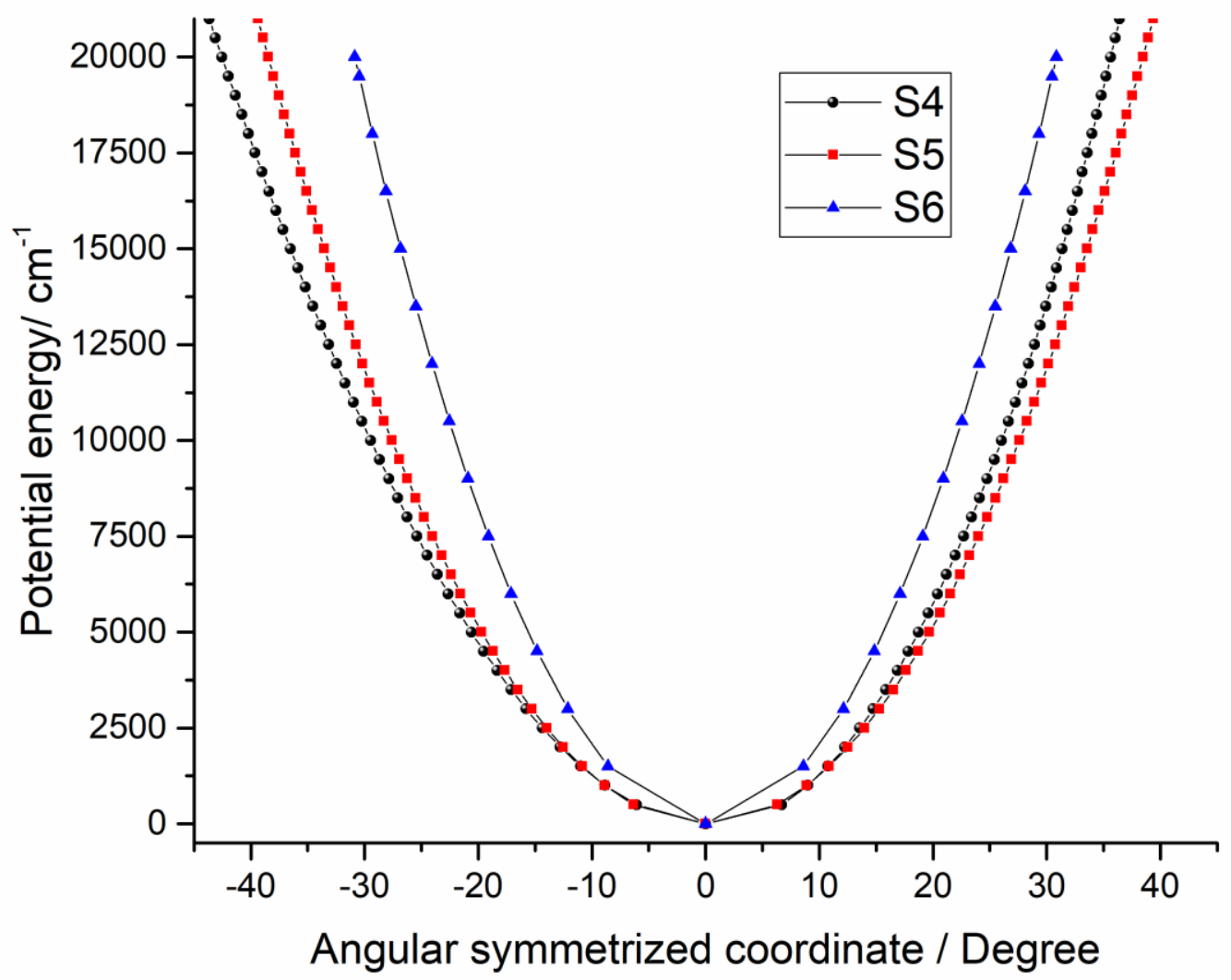

Figure 2. One-dimensional PES sections for angular symmetrized coordinates. benchmark PES calculations were carried out using the least-squares fit to V5Z grid values, 
without the further corrections discussed in the following sections. Even though the V5Z basis set did not provide the best quantitative accuracy, we considered that it was preferable to use all PESs at the same theoretical level without corrections for a reliable comparison of the general qualitative shape of the surfaces. A $b$ initio potential energies were fitted using the analytical symmetry adapted representation (3) and the weight function employed by Schwenke and Partridge in ref [58]

$$
w(E)=\frac{\tanh \left(-0.0005\left(E-E_{1}\right)+1.002002002\right)}{2.002002002} .
$$

This weight function decreases with the energy $E$ (expressed in $\mathrm{cm}^{-1}$ ) in order to de-emphasize the contribution to the PES fit of large grid displacements for vibrational energies beyond $E_{l}=15000 \mathrm{~cm}^{-1}$. The least-squares fit of the PES in various sets of coordinates leads to quite similar results, but for the coordinate sets II and III, the RMS deviations slightly decrease at $E_{1}>$ $12000 \mathrm{~cm}^{-1}$. Table 2 summarizes the RMS deviations of the fit in various coordinates. The PES expansion was extended up to sixth order in all $S_{i}$-coordinates including the torsion function $\underline{\cos (\tau)+1}$ but some eighth-order terms were added for torsion functions $\underline{\cos (\tau / 2) \text { and } \tau-\pi}$.

The number of parameters, which were statistically well determined in the fit of the PES to ab initio electronic energies, versus the total number of parameters of the corresponding expansion orders, is given in the second row of Table 2. The results show that the coordinate sets (II) and (III) provide a more accurate description of the PES when using the weight function (3). When fitting a six-dimensional PES, all three forms of the torsion function result in close lying standard deviation (STD) values but the use of (II) and (III) provides slightly lower RMS values. Ref [14] reported a more complicated PES representation, but the use of that representation was not found useful in our case.

Table 2. RMS and standard deviations of the PES fits in various coordinate sets.

\begin{tabular}{|c|c|c|c|c|c|}
\hline $\begin{array}{c}\text { Coordinates } \\
\text { PES, \#Calc, }\end{array}$ & $\begin{array}{c}\text { (I) Internal } \\
\text { Fig. 1 (a), } \\
\text { Calc 1 }\end{array}$ & $\begin{array}{c}\text { (I) Internal } \\
\text { Fig. 1 (a), } \\
\text { Calc 0 }\end{array}$ & $\begin{array}{c}\text { (I) Internal } \\
\text { Fig. 1 (a), } \\
\text { Calc 4 }\end{array}$ & $\begin{array}{c}\text { (II) O-X } \\
\mathrm{X}\left(\mathrm{CH}_{1} \mathrm{H}_{2}\right) \\
\text { Fig. 1 (b), } \\
\text { Calc 2 }\end{array}$ & $\begin{array}{c}\text { (III) } \\
\text { Orthogonal } \\
\text { Fig. 1 (c), } \\
\text { Calc 3 }\end{array}$ \\
\hline $\begin{array}{c}\text { Torsion } \\
\text { function }\end{array}$ & $\underline{\cos (\tau)+1}$ & $\underline{\cos (\tau / 2)}$ & $\underline{\tau-\pi}$ & $\underline{\cos (\tau / 2)}$ & $\underline{\cos (\tau / 2)}$ \\
\hline $\begin{array}{c}\text { \#Parameters/ } \\
\text { Initial \# } \\
\text { Param }\end{array}$ & $342 / 502$ & $344 / 576$ & $338 / 576$ & $358 / 576$ & $356 / 576$ \\
\hline KEO & Exact & Exact & Watson & Exact & Exact \\
\hline RMS (STD) & $1.58(1.25)$ & $1.99(1.27)$ & $1.59(1.24)$ & $1.09(0.84)$ & $1.14(0.84)$ \\
\hline
\end{tabular}

Note: Abbreviation KEO stands for kinetic energy operator 
To control the equilibrium geometry, we calculated the rotational energy levels combining the V5Z PES with the equilibrium geometries optimized using larger basis sets. This corresponds to a series of calculations using the aug-cc-pCVXZ core-valence basis sets augmented with diffuse functions for one-particle basis cardinal numbers $\mathrm{X}=4$ and 5 (denoted simply as ACVQZ, and ACV5Z). We have also accounted for the Douglas-Kroll (DK) type relativistic corrections. The parameters of the equilibrium geometry affect the vibrational energy levels, especially those associated with the angular motions_[55]. Table 3 gives the equilibrium geometry parameters, while Table 4 gives the energy levels at $\mathrm{J}=1$ for the equilibrium geometries mentioned in Table 3. Note that changes up to the fourth decimal place do not affect the vibrational energy levels. That is why, the calculations for all geometries except for the first one, led to almost identical results for the low lying rotational levels. The accuracy of the known experimental rotational levels was estimated to be about $10^{-4} \mathrm{~cm}^{-1}$. In this work, however, we did not have an intention to obtain highly accurate values of the rotational levels, but to find the appropriate parameters of the equilibrium geometry consistent with reliable vibrational predictions.

Table 3. Equilibrium geometry of the formaldehyde molecule, as optimized at various levels of ab initio theory

\begin{tabular}{|c|c|c|c|c|c|}
\hline Coordinates & $\begin{array}{l}\text { CCSD(T)/ } \\
\text { ACVQZ }\end{array}$ & $\begin{array}{l}\text { CCSD(T)/ } \\
\text { ACV5Z }\end{array}$ & $\begin{array}{l}\text { CCSD(T)-DK/ } \\
\text { ACV5Z-DK** }\end{array}$ & Morgan [13] & $\begin{array}{l}\text { Empirical } \\
\text { values [59] [60] }\end{array}$ \\
\hline $\mathrm{r}_{\mathrm{e}}(\mathrm{CO}) / \AA$ & 1.205240 & 1.20437 & 1.20426 & 1.20457 & $1.20461(19)$ \\
\hline $\mathrm{r}_{\mathrm{e}}(\mathrm{CH}) / \AA$ & 1.100744 & 1.10041 & 1.10031 & 1.10052 & $1.10046(16)$ \\
\hline$\theta(\mathrm{HCH}) / \mathrm{Deg}$ & 116.6186 & 116.6495 & 116.6546 & 116.694 & $116.722(93)$ \\
\hline$\theta(\mathrm{OCH})^{*} / \mathrm{Deg}$ & 121.6906 & 121.6752 & 121.6726 & 121.653 & 121.639 \\
\hline
\end{tabular}

$* \underline{\theta(\mathrm{OCH})}=\pi-\theta(\mathrm{HCH}) / 2$

** accounting for Douglas-Kroll-Hess relativistic corrections

Table 4. The energy levels (in $\mathrm{cm}^{-1}$ ) obtained for the equilibrium geometries from Table 3 with the (V5Z) PES.

\begin{tabular}{|l|l|l|l|l|l|}
\hline J=1 Levels & Exp.* & ACV5Z** & ACV5Z** & $\begin{array}{l}\text { Morgan } \\
{[\mathbf{1 3}]}\end{array}$ & Empirical \\
"Exact” KEO & vatson KEO & values [59] [60] \\
\hline $\mathrm{A}_{2}$ & 2.4295 & 2.422452 & 2.426531 & 2.421981 & 2.422041 \\
\hline $\mathrm{B}_{1}$ & 10.5395 & 10.539508 & 10.537128 & 10.533067 & 10.531232 \\
\hline
\end{tabular}




\begin{tabular}{|l|l|l|l|l|l|}
\hline $\mathrm{B}_{2}$ & 10.7006 & 10.699928 & 10.702374 & 10.693525 & 10.691728 \\
\hline
\end{tabular}

** Basis sets used for the equilibrium geometry. KEO stands for the kinetic energy operator used in the calculations

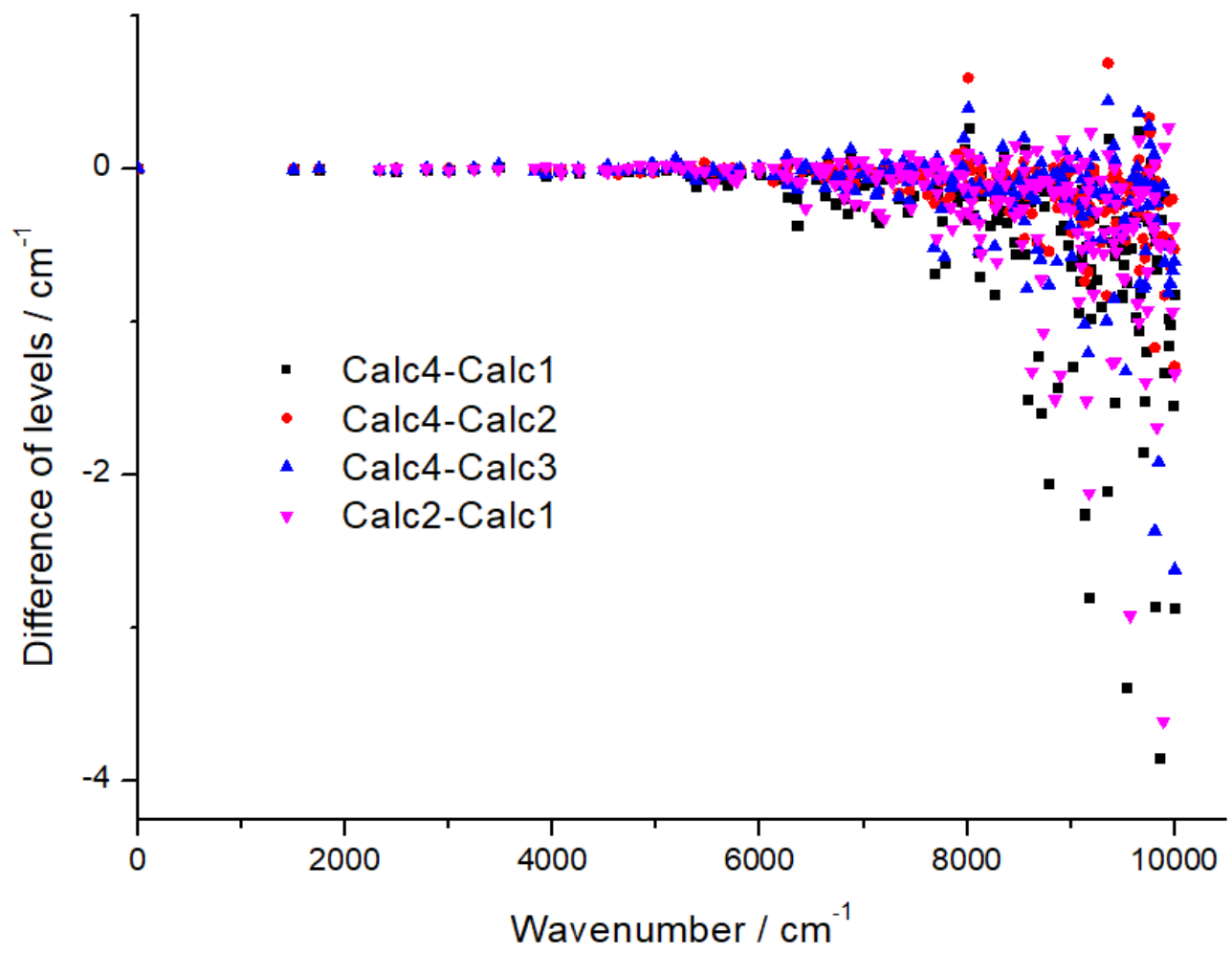

Figure 3. Comparison of the energy levels (the symmetry block $A_{1}$ ) for four calculations. The variational methods and the ab initio PES versions corresponding to Calc1, Calc2, Calc3 and Calc4 are defined in Table 2.

Figure 3 displays the diagram of differences in the calculated levels for the symmetry block $\mathrm{A}_{1}$. The diagram shows that the difference between the fourth and the first calculation is considerable in the range over $8000 \mathrm{~cm}^{-1}$. This is mainly caused by the different torsion parts. Below $7000 \mathrm{~cm}^{-1}$, all calculations lead to close results. Below $4500 \mathrm{~cm}^{-1}$, the influence of the PES type on the energy levels is not significant: the differences in the level positions do not exceed $0.05 \mathrm{~cm}^{-1}$.

\section{Improvement of the basis set and corrections to the PES}

Since all versions of calculations for levels up to $7000 \mathrm{~cm}^{-1}$ using the V5Z basis in the previous sections lead to close results, we shall use the internal coordinate set (I) and torsion function $\cos (\tau / 2)$ while improving the PES at higher levels of the theory. The choice of the shape is due to the fact that the internal coordinates are easier for calculating corrections.

First of all, we conducted calculations according to the procedure described for the methane molecule in ref [12] using the augmented core-valence basis set ACV5Z at a sparser grid of 
geometries. As these calculations are more demanding, we computed electronic energies with the $\operatorname{CCSD}(\mathrm{T}) / \mathrm{ACV} 5 \mathrm{Z}$ level of theory at the grid $\left(\mathrm{G}^{(\mathrm{AC})}\right.$ ) containing only 3820 points in the range up to about $6000 \mathrm{~cm}^{-1}$ from the equilibrium geometry. Similarly to ref [12], the energy differences $\Delta_{\mathrm{AC}}=$ ACV5Z-V5Z involving two basis sets were computed on this grid. We have checked that these differences behave quite smoothly with respect to the symmetrized coordinates $S_{i}$ and were possible to be modeled analytically using the fourth-order expansions $\Delta_{\mathrm{AC}}\left(S_{i}\right)$ of the type (2a2c). This allowed us to extend the electronic energies according to the relation ACV5Z $\approx V 5 Z+$ $\Delta_{\mathrm{AC}}\left(S_{i}\right)$ for all remaining geometries and refitting the PES(ACV5Z) on the full reference grid $\mathrm{G}^{(\mathrm{R})}$ of 22509 points using the analytical form (2a) as discussed in the previous section.

As the next step, we accounted for the following three corrections to the surface: "massvelocity-Darwin" (MVD) relativistic corrections, the diagonal Born-Oppenheimer correction (DBOC), and higher-order dynamic electron correlations (HODEC). The adiabatic DBOC corrections [62] [63] [64] [65] [66], which are the leading first-order contributions to the electronic energy beyond the Born-Oppenheimer approximation have been included in accurate PES calculations for several small molecules (see refs. [67] [68] [21] [38] and references therein), however, to our knowledge have not yet been considered so far for formaldehyde. It has been argued in many previous works [69] [70] [71] [72] [73] [74] that dynamic electron correlations beyond single and double excitations are important to approach the spectroscopic accuracy. They have been included in recent spectroscopically accurate PESs of methane [12] [75], whereas Morgan et al. [13] have reported a very thorough study of the impact of triple and quadruple excitations to the equilibrium geometry and the quartic force field of formaldehyde.

In this work, we calculated MVD corrections with MOLPRO on the full reference grid $G^{(R)}$ 302 of 22509 points. Much more expensive calculations for DBOC and HODEC corrections were carried out at 2000 points of a smaller grid $\mathrm{G}^{\text {(Corr) }}$ of nuclear geometries and approximated by a $4^{\text {th }}$ order expansion of the type (2). This gave a quite smooth analytical function of all six symmetrized coordinates for the sum of these three corrections:

$$
\Delta_{(3 \text { corr })}\left(S_{i}\right)=\Delta_{\mathrm{Rel}}\left(S_{i}\right)+\Delta_{\mathrm{DBOC}}\left(S_{i}\right)+\Delta_{\mathrm{T}(\mathrm{Q})}\left(S_{i}\right) .
$$

The DBOC and HODEC corrections were calculated using the CFOUR program suite [76] with the cc-pVTZ one-particle basis set. The HODEC corrections on the $\mathrm{G}^{\text {(Corr) }}$ grid were computed using the noniterative quadruple $\operatorname{CCSDT}(\mathrm{Q})$ method [71] [72] [73] [74]. At this step we have constructed the $a b$ initio corrected surface

$$
\operatorname{PES}(\mathrm{ACV} 5 \mathrm{Z}+3 \text { Corr })=\operatorname{PES}(\mathrm{ACV} 5 \mathrm{Z})+\Delta_{(3 \text { corr })}\left(S_{i}\right)
$$

Table 5 gives the energy levels using the PES(ACV5Z) and PES(ACV5Z+3Corr) that account for the above considered corrections, analytically extrapolated from the sparser grid $\mathrm{G}^{\text {(Corr) }}$. 
It is seen that a values of the HODEC dynamic correction for formaldehyde computed with the $\operatorname{CCSDT}(\mathrm{Q})$ method is several times larger than those for the methane molecule [12] . The differences between the calculated and measured levels are considerably larger for the $v_{2}$ overtones. Although the PES (ACV5Z + 3Corr) for formaldehyde is constructed in a similar way as the PES for methane, its accuracy is significantly lower than that for methane. It was necessary to improve the potential energy surface, particularly along the CO bond.

Since the relativistic corrections and DBOC are relatively small, we have tried to explore further contributions related to HODEC correlations including quadruple excitations, as well as to the larger, aug-cc-pCV6Z basis set. To this end we used at the next step the iterative CCSDTQ [73] [74] [76] method which is expected to provide more rigorous account for dynamic electronic correlations corresponding to quadruple excitations. However, this method converged very slowly for low-symmetry geometries or for large $\mathrm{r}_{\mathrm{CO}}$ bond distances. Full PES calculations at this level of the theory are extremely demanding, but it was possible to study the corresponding contributions on the most relevant one-dimensional cuts. Previous experiences in the PES corrections for various molecules showed that corrections performed along chemical bonds provide a maximum contribution. Note that for formaldehyde, all geometries keep the $\mathrm{C}_{2 \mathrm{v}}$ symmetry while changing the $r_{C O}$ bond length, whereas they have the $C_{S}$ symmetry while changing the $\mathrm{r}_{\mathrm{CH}}$ bond length. The correction $\Delta_{\mathrm{Q}}=\operatorname{CCSDTQ}$ - CCSDT(Q) was evaluated for about twenty points along both bond stretching coordinates. The one-dimensional $\Delta_{\mathrm{AC} 6 \mathrm{Z}}=$ ACV6Z-ACV5Z corrections were also computed at the same sets of geometries. The onedimensional corrections of the $\mathrm{CO}$ cut of the potential are shown in Figure 4. These corrections result in an increase of the potential energy at distances longer than $\underline{\mathrm{r}_{\mathrm{e}}}(\mathrm{CO})$. At the distances shorter than $\underline{r}_{2}(\mathrm{CO})$, the potential energy slightly decreases. For the $\mathrm{r}_{\mathrm{CO}}$ bond, the $\Delta_{\mathrm{Q}}=$ CCSDTQCCSDT(Q) correction in the VTZ basis considerably exceeds the $\Delta_{\mathrm{AC6Z}}=$ ACV6Z-ACV5Z correction calculated with the $\operatorname{CCSD}(\mathrm{T})$ technique. For the $\mathrm{r}_{\mathrm{CH}}$ bond, the contributions of these corrections are of a similar magnitude. We summarized two corrections $\Delta_{\mathrm{Q}+6 \mathrm{Z}}=\Delta_{\mathrm{Q}}+\Delta_{\mathrm{AC} 6 \mathrm{Z}}$ and approximated it analytically as a power series expansion in Morse-type functions $\Delta_{\mathrm{Q}+6 \mathrm{Z}}\left(S_{1}, S_{2}, S_{5}\right)$ of radial coordinates (2b). The choice of analytical representations had a marginal effect on vibrational energy levels.

Our final ab intio PES including these four corrections was constructed as

$$
\operatorname{PES}(\mathrm{ACV} 5 Z+4 \text { Corr })=\operatorname{PES}(\mathrm{ACV} 5 Z)+\Delta_{(3 \mathrm{corr})}\left(S_{i}\right)+\Delta_{\mathrm{Q}+6 \mathrm{Z}}\left(S_{1}, S_{2}, S_{5}\right)
$$

The fourth correction $\Delta_{\mathrm{Q}+6 \mathrm{Z}}$ improves the accuracy of the calculated fundamental frequencies and brings them closer to the measured values, especially for $v_{2}$. Also, the correction diminished the calculated value of the equilibrium geometry $r_{\mathrm{CO}}$. Contrary to the $r_{\mathrm{CO}}$ correction, the $\mathrm{r}_{\mathrm{CH}}$ correction does have a significant effect on the energy levels. The partial contributions of the 
350 Figure 6.

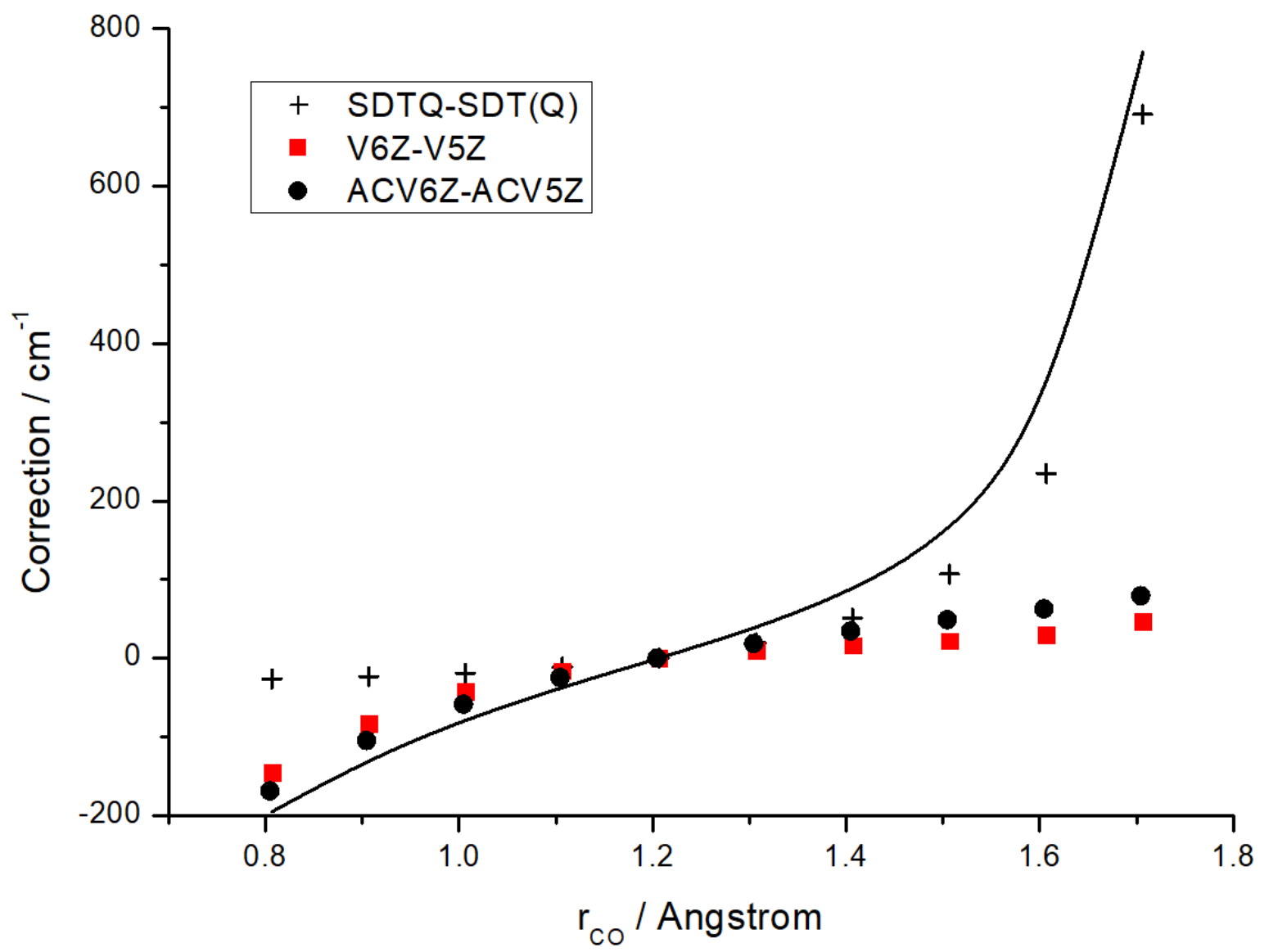

Figure 4 One-dimensional radial corrections along the $C O$ bond(shown as solid line). The contributions $\Delta_{Q}=C C S D T Q-C C S D T(Q)$ - the rigorous account for connected quadruple excitations in dynamic electron correlation - are shown as black crosses, while the $\Delta_{A C 6 Z}=(A C V 6 Z-A C V 5 Z)$ and $\Delta_{V 6 Z}=$ (V6Z-V5Z) corrections calculated at the CCSD(T) level are shown as circles and squares, respectively. The sum of two corrections $\Delta_{Q+6 Z}=\Delta_{Q}+\Delta_{A C 6 Z}$ shown as a solid line. 


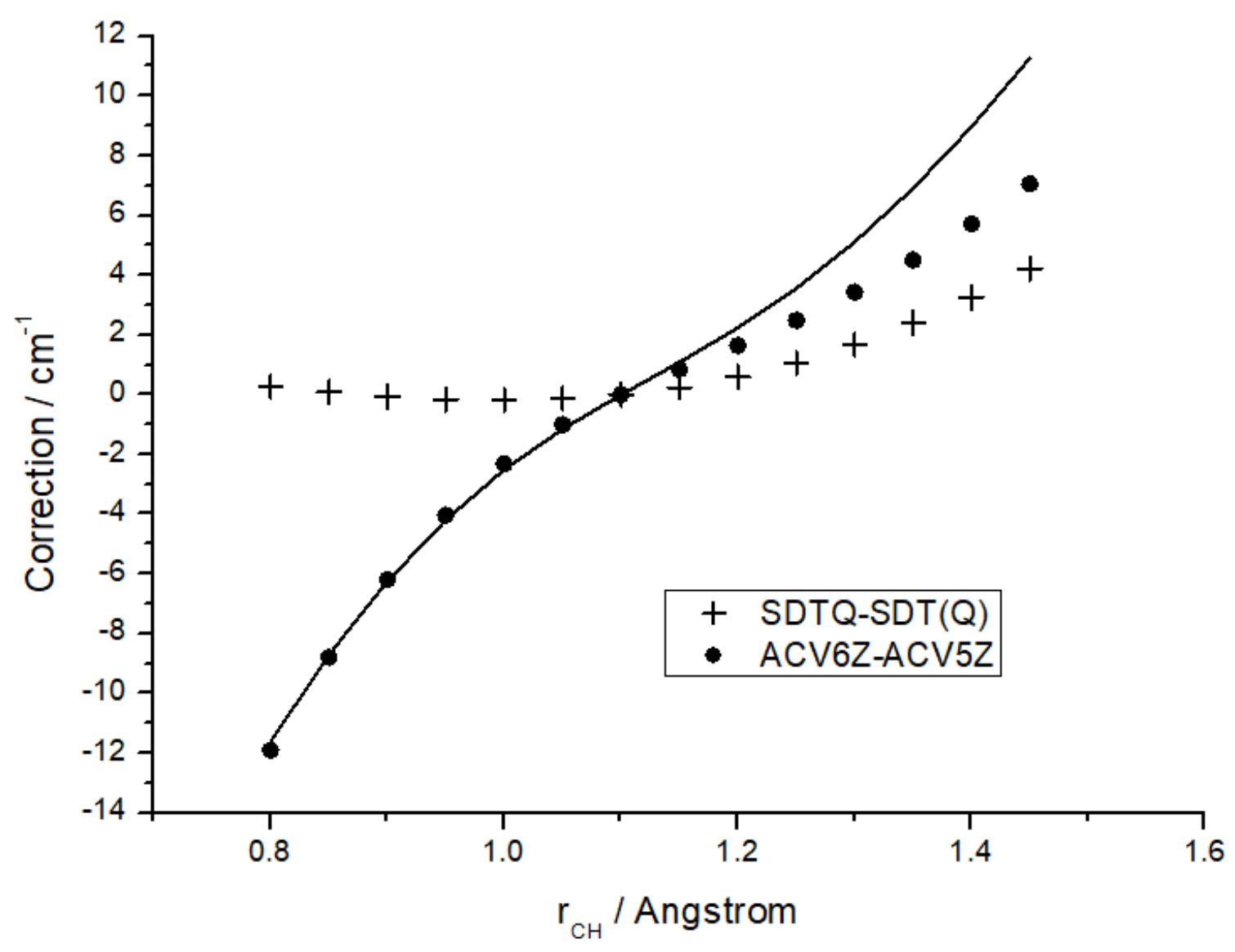

Figure 5 One-dimensional radial corrections along the $\mathrm{CH}$ bond (shown as solid line). The contributions $\Delta_{Q}=$ CCSDTQ - CCSDT(Q) - the rigorous account for connected quadruple excitations in dynamic electron correlation - are shown as black crosses, while the $\triangle_{A C 6 Z}=(A C V 6 Z-A C V 5 Z)$ correction calculated at the $\operatorname{CCSD}(T)$ level is shown as circles. The sum of two corrections $\Delta_{Q+6 Z}=\Delta_{Q}+\Delta_{A C 6 z}$ shown as a solid line.

The contributions of various corrections to vibrational levels and our final ab initio results are collected in Table 5. The experimental energy levels are taken from refs [77] [78] [79], and [80]. Note that the (Exp.-Calc.) deviations exceed $1 \mathrm{~cm}^{-1}$ only for six levels among a total of 35 experimentally known levels. The deviation of $-1 \mathrm{~cm}^{-1}$ for $2 v_{2}$ is quite reasonable considering that the deviation for $v_{2}$ is equal to $-0.44 \mathrm{~cm}^{-1}$. Four levels from the other five have been taken from ref [80]. This work reported the level values with only one significant digit after the decimal point. Ref [79] reported another vibrational band center $v_{2}+v_{4}+v_{6}$ derived from 40 rotational-vibrational energy levels including $\mathrm{J}$ values up to 17 with an uncertainty of $0.33 \mathrm{~cm}^{-1}$. Unfortunately, this work [79] did not mention a minimum value of $\mathrm{J}$ for experimental transitions in the $v_{2}+v_{4}+v_{6}$ band. If the fitting procedure does not use a sufficient number of the assigned transitions with $\mathrm{J}<6$, the accuracy of the extrapolated empirical $\mathrm{J}=0$ level might be poor. $\mathrm{A}$ large difference can be explained by the fact that the two calculations deviate in different directions. Four other band centers above $4400 \mathrm{~cm}^{-1}$ have been reported in ref [79]: $v_{1}+v_{2} \underline{\text { at }}$

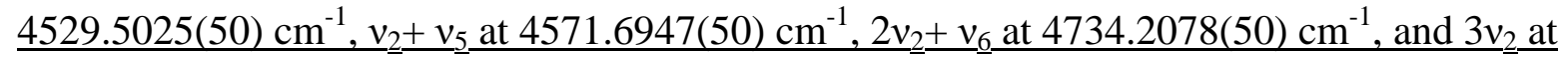


overestimation is due to the deviation of $v_{2}$ levels, which can be roughly estimated as $-0.44 \mathrm{n}$

Table 5. Vibrational levels with contributions of relativistic, DBOC corrections, and high-order electron correlations up to $4400 \mathrm{~cm}^{-1}$.

\begin{tabular}{|c|c|c|c|c|c|c|c|c|c|c|c|c|}
\hline \multirow{3}{*}{ Vib. State } & \multirow{3}{*}{$\mathrm{C}$} & \multirow{3}{*}{$\begin{array}{l}\text { Exp. } \\
\text { levels* }\end{array}$} & \multicolumn{8}{|c|}{ This work } & \multicolumn{2}{|c|}{ ref. [14] } \\
\hline & & & \multicolumn{4}{|c|}{$\begin{array}{l}\text { Contributions of } \\
\text { corrections }\end{array}$} & \multicolumn{4}{|c|}{ Theoretical levels with corrections } & \multirow[t]{2}{*}{ Calc } & \multirow{2}{*}{$\begin{array}{l}\text { Exp- } \\
\text { Calc }\end{array}$} \\
\hline & & & Rel. & $\begin{array}{l}\text { DBO } \\
\mathrm{C}\end{array}$ & $T(Q)$ & $Q+6 Z$ & ACV5Z & $\begin{array}{l}\text { ACV5Z } \\
+3 \text { corr }\end{array}$ & $\begin{array}{l}\text { ACV5Z } \\
+4 \text { corr }\end{array}$ & $\begin{array}{c}\text { Exp- } \\
\text { Calc } \\
\# \#\end{array}$ & & \\
\hline$v_{4}$ & $\mathrm{~B}_{1}$ & $1167.25628(2)^{\mathrm{a}}$ & -0.14 & -0.07 & -3.82 & 0.55 & 1170.469 & 1166.43 & 1166.99 & 0.27 & 1166.1 & 1.16 \\
\hline$v_{6}$ & $\mathrm{~B}_{2}$ & $1249.09568(2)^{\mathrm{a}}$ & -0.06 & 0.08 & -2.24 & 0.65 & 1250.471 & 1248.25 & 1248.91 & 0.18 & 1245.6 & 3.49 \\
\hline$v_{3}$ & $\mathrm{~A}_{1}$ & $1500.17574(12)^{\mathrm{a}}$ & 0.06 & -0.13 & -2.28 & 0.76 & 1501.967 & 1499.62 & 1500.38 & -0.21 & 1499.1 & 1.07 \\
\hline$v_{2}$ & $\mathrm{~A}_{1}$ & $1746.00886(13)^{\mathrm{a}}$ & -0.91 & -0.23 & -8.86 & 3.75 & 1752.703 & 1742.69 & 1746.45 & -0.44 & 1744.61 & 1.4 \\
\hline $2 v_{4}$ & $\mathrm{~A}_{1}$ & $2327.5239(5)^{b}$ & -0.28 & -0.14 & -7.59 & 1.10 & 2333.903 & 2325.89 & 2326.99 & 0.53 & 2325.18 & 2.35 \\
\hline$v_{4}+v_{6}$ & $\mathrm{~A}_{2}$ & $2422.9701(50)^{\mathrm{b}}$ & -0.21 & 0.00 & -6.10 & 1.22 & 2427.594 & 2421.29 & 2422.52 & 0.45 & 2418.43 & 4.54 \\
\hline $2 v_{6}$ & $\mathrm{~A}_{1}$ & $2494.3543(5)^{\mathrm{b}}$ & -0.13 & 0.15 & -4.49 & 1.32 & 2497.191 & 2492.71 & 2494.03 & 0.32 & 2487.73 & 6.63 \\
\hline$v_{3}+v_{4}$ & $\mathrm{~B}_{1}$ & $2667.0481(20)^{\mathrm{b}}$ & -0.09 & -0.19 & -6.18 & 1.33 & 2672.132 & 2665.67 & 2667.00 & 0.05 & 2664.64 & 2.4 \\
\hline$v_{3}+v_{6}$ & $\mathrm{~B}_{2}$ & $2719.1550(5)^{b}$ & -0.03 & -0.15 & -4.43 & 1.04 & 2722.697 & 2718.08 & 2719.13 & 0.03 & 2715.24 & 3.92 \\
\hline$v_{1}$ & $\mathrm{~A}_{1}$ & $2782.4575(5)^{b}$ & -0.25 & -0.61 & -4.78 & 0.34 & 2788.101 & 2782.46 & 2782.81 & -0.35 & 2781.74 & 0.71 \\
\hline$v_{5}$ & $\mathrm{~B}_{2}$ & $2843.3256(5)^{b}$ & -0.37 & -0.37 & -6.14 & 0.90 & 2848.961 & 2842.08 & 2842.98 & 0.34 & 2842.37 & 0.95 \\
\hline$v_{2}+v_{4}$ & $\mathrm{~B}_{1}$ & $2905.9685(20)^{b}$ & -1.06 & -0.30 & -12.8 & 4.34 & 2916.043 & 2901.80 & 2906.14 & -0.18 & 2903.35 & 2.61 \\
\hline $2 v_{3}$ & $\mathrm{~A}_{1}$ & $2998.9873(5)^{b}$ & 0.11 & -0.25 & -4.69 & 1.55 & 3002.723 & 2997.89 & 2999.44 & -0.46 & 2997.24 & 1.74 \\
\hline$v_{2}+v_{6}$ & $\mathrm{~B}_{2}$ & $3000.0659(5)^{b}$ & -0.88 & -0.19 & -10.4 & 3.98 & 3007.897 & 2996.34 & 3000.32 & -0.26 & 2995.91 & 4.16 \\
\hline$v_{2}+v_{3}$ & $\mathrm{~A}_{1}$ & $3238.4548(20)^{\mathrm{b}}$ & -0.89 & -0.36 & -11.3 & 4.62 & 3247.114 & 3234.49 & 3239.10 & -0.65 & 3236.11 & 2.35 \\
\hline $2 v_{2}$ & $\mathrm{~A}_{1}$ & 3471.6 & -1.81 & -0.46 & -17.8 & 7.58 & 3485.266 & 3465.10 & 3472.69 & -1.09 & 3469.39 & 2.21 \\
\hline $3 v_{4}$ & $\mathrm{~B}_{1}$ & 3480.7 & -0.41 & -0.21 & -11.3 & 1.65 & 3491.059 & 3479.11 & 3480.76 & -0.06 & 3478.04 & 2.66 \\
\hline $2 v_{4}+v_{6}$ & $\mathrm{~B}_{2}$ & 3586.6 & -0.35 & -0.06 & -9.82 & 1.77 & 3594.654 & 3584.42 & 3586.19 & 0.41 & 3581.09 & 5.51 \\
\hline$v_{4}+2 v_{6}$ & $\mathrm{~B}_{1}$ & $3673.5^{\$}$ & -0.28 & 0.07 & -8.42 & 1.86 & 3682.802 & 3674.16 & 3676.03 & -2.53 & 3668.88 & 4.62 \\
\hline $3 v_{6}$ & $\mathrm{~B}_{2}$ & - & -0.21 & 0.20 & -6.79 & 1.89 & 3740.452 & 3733.65 & 3735.54 & - & - & - \\
\hline$v_{3}+2 v_{4}$ & $\mathrm{~A}_{1}$ & 3825.3 & -0.24 & -0.26 & -9.98 & 1.89 & 3834.334 & 3823.86 & 3825.74 & -0.45 & 3822.43 & 2.87 \\
\hline$v_{3}+v_{4}+v_{6}$ & $\mathrm{~A}_{2}$ & $3886.5^{\$}$ & -0.21 & -0.25 & -8.39 & 1.50 & 3895.439 & 3886.59 & 3888.10 & -1.60 & 3883.37 & 3.13 \\
\hline$v_{3}+2 v_{6}$ & $\mathrm{~A}_{1}$ & $3937.4^{\$}$ & -0.14 & -0.14 & -6.77 & 1.49 & 3940.654 & 3933.60 & 3935.09 & 2.31 & 3929.86 & 7.54 \\
\hline$v_{1}+v_{4}$ & $\mathrm{~B}_{1}$ & $3941.5295^{\mathrm{c}}$ & -0.40 & -0.66 & -8.83 & 0.89 & 3950.568 & 3940.68 & 3941.57 & -0.04 & 3939.85 & 1.68 \\
\hline$v_{4}+v_{5}$ & $\mathrm{~A}_{2}$ & $3996.518^{c}$ & -0.49 & -0.40 & -9.94 & 1.48 & 4005.302 & 3994.47 & 3995.94 & 0.57 & 3994.75 & 1.77 \\
\hline$v_{1}+v_{6}$ & $\mathrm{~B}_{2}$ & $4021.0806(6)^{\mathrm{d}}$ & -0.31 & -0.50 & -7.17 & 1.00 & 4028.157 & 4020.18 & 4021.18 & -0.10 & 4017.7 & 3.38 \\
\hline$v_{2}+2 v_{4}$ & $\mathrm{~A}_{1}$ & 4058.3 & -1.18 & -0.36 & -16.5 & 4.84 & 4071.474 & 4053.43 & 4058.28 & 0.02 & 4054.6 & 3.7 \\
\hline$v_{5}+v_{6}$ & $\mathrm{~A}_{1}$ & 4083.1 & -0.52 & -0.21 & -9.30 & 2.07 & 4090.512 & 4080.47 & 4082.54 & 0.56 & 4078.88 & 4.22 \\
\hline$v_{2}+v_{4}+v_{6}$ & $\mathrm{~A}_{2}$ & $4163.289(330)^{\mathrm{d}, \$}$ & -1.06 & -0.26 & -14.6 & 4.66 & 4176.049 & 4160.08 & 4164.74 & -1.45 & 4159.35 & 3.94 \\
\hline $2 v_{3}+v_{4}$ & $\mathrm{~B}_{1}$ & - & -0.05 & -0.31 & -8.65 & 2.13 & 4172.264 & 4163.25 & 4165.37 & - & - & - \\
\hline $2 v_{3}+v_{6}$ & $\mathrm{~B}_{2}$ & $4192.3816(5)^{d}$ & 0.00 & -0.33 & -6.74 & 1.54 & 4198.047 & 4190.96 & 4192.51 & -0.13 & 4189.62 & 2.77 \\
\hline$v_{2}+2 v_{6}$ & $\mathrm{~A}_{1}$ & 4248.7 & -0.87 & -0.15 & -12.2 & 4.27 & 4256.837 & 4243.52 & 4247.79 & 0.90 & 4241.02 & 7.68 \\
\hline$v_{1}+v_{3}$ & $\mathrm{~A}_{1}$ & $4253.8^{\$}$ & -0.18 & -0.72 & -7.00 & 1.07 & 4262.684 & 4254.79 & 4255.86 & -2.06 & 4253.86 & -0.06 \\
\hline
\end{tabular}




\begin{tabular}{|c|c|c|c|c|c|c|c|c|c|c|c|c|}
\hline$v_{3}+v_{5}$ & $\mathrm{~B}_{2}$ & $4335.0971(6)^{\mathrm{d}}$ & -0.30 & -0.42 & -8.69 & 1.90 & 4342.566 & 4333.15 & 4335.05 & 0.05 & 4334.78 & 0.32 \\
\hline$v_{2}+v_{3}+v_{4}$ & $\mathrm{~B}_{1}$ & 4397.5 & -1.05 & -0.43 & -15.4 & 5.22 & 4409.877 & 4392.92 & 4398.14 & -0.64 & 4394.05 & 3.45 \\
\hline RMS deviation & \\
RMS (-5 lev.)
\end{tabular}

*) Experimental values given in wavenumber units $\left(\mathrm{cm}^{-1}\right)$, a - from ref [81], b - from ref [78], c - from ref [77], $d-$ from ref [79], with one decimal place from ref [80].

\#) Rel.= relativistic; $T(Q)=\operatorname{CCSDT}(\mathrm{Q})-\operatorname{CCSD}(\mathrm{T})$ correlation; $\mathrm{Q}+6 Z$ = quadruple $\Delta_{\mathrm{Q}+6 \mathrm{Z}}$ correction (see text for explanation).

\#\#) Experimental minus calculated levels corresponding to the full ab initio PES(ACVZ6 + 4Corr).

\$) RMS without five suspicious levels given in Table 6.

Note that most of the "outlier" levels in our (exp.-calc.) with deviations larger than $1 \mathrm{~cm}^{-1}$ also showed significant deviations from empirical values in calculations of Burleigh et al. [15] and of Yachmenev et al. [14]. Table 6 compares three calculations for these five levels.

Table 6. Five vibrational levels from Table 5 with our (Exp.-Calc.) deviations exceeding $1 \mathrm{~cm}^{-1}$.

\begin{tabular}{|c|c|c|c|c|c|c|c|c|}
\hline Vib. State & C & Exp. * & \multicolumn{2}{|c|}{ This work } & \multicolumn{2}{c|}{ ref. [15] } & \multicolumn{2}{c|}{ ref. [14] } \\
\hline$v_{4}+2 v_{6}$ & $\mathrm{~B}_{1}$ & $\mathbf{3 6 7 3 . 5}^{?}$ & 3676.032 & -2.53 & 3675.9 & -2.4 & 3675.07 & -1.57 \\
\hline$v_{3}+v_{4}+v_{6}$ & $\mathrm{~A}_{2}$ & $\mathbf{3 8 8 6 . 5}^{?}$ & 3888.101 & -1.60 & 3886.7 & -0.2 & 3886.47 & 0.03 \\
\hline$v_{3}+2 v_{6}$ & $\mathrm{~A}_{1}$ & $\mathbf{3 9 3 7 . 4}^{?}$ & 3935.095 & 2.31 & 3935.4 & 2.0 & 3936.02 & 1.38 \\
\hline$v_{2}+v_{4}+v_{6}$ & $\mathrm{~A}_{2}$ & $\mathbf{4 1 6 3 . 2 8 9} ?$ & 4164.739 & -1.45 & 4163.6 & 0.3 & 4164.12 & -0.83 \\
\hline$v_{1}+v_{3}$ & $\mathrm{~A}_{1}$ & $\mathbf{4 2 5 3 . 8}$ & 4255.859 & -2.06 & 4255.3 & -1.5 & 4254.58 & -0.78 \\
\hline
\end{tabular}

*) experimental values reported in refs [80], [79]

Note that empirical levels reported by Bouwens et al. [80] in 1996 have been obtained from low resolution dispersed fluorescence spectra with an average uncertainty of $1 \mathrm{~cm}^{-1}$. However, some of these empirical levels could have larger errors as they differ considerably from values obtained from high-resolution spectra analyses in more recent papers. For example, the study of Bouwens et al [80] reported the $v_{3}+v_{6}$ level at $2496.11 \mathrm{~cm}^{-1}$ and the $v_{1}+v_{4}$ level at $3940.21 \mathrm{~cm}^{-}$

$404{ }^{1}$. In a more recent paper of A. Perin et al. [78] [81] the same levels were obtained from analyses 405 of vibration-rotation spectra at $2494.3543 \mathrm{~cm}^{-1}$ and at $3941.5295 \mathrm{~cm}^{-1}$, respectively. Figure 7 406 shows the diagram illustrating the differences between empirical levels obtained from dispersed 407 fluorescence spectra [80] and those obtained from infrared rotationally resolved spectra [78] [81]. A comparison of fundamental band centers computed from our final ab initio PES including all corrections with the results of the recent, very thorough study by Morgan et al [13]

410 is given in Table 7. According to notations of ref [13], here MAE $\left(\frac{1}{n} \sum_{i=1}^{n}\left|X_{i}-Y_{i}\right|\right)$ is the mean 
411 (Exp.-Calc.) deviation, while MAPE $\left(\frac{100 \%}{n} \sum_{i=1}^{n}\left|\frac{X_{i}-Y_{i}}{X_{i}}\right|\right)$ is the mean absolute percent error 412 defined by Eq.(17) of ref [13].

413

414

Table 7. Experimental minus calculated deviations for fundamentals of $\mathrm{H}_{2} \mathrm{CO}$.

\begin{tabular}{|c|c|c|c|c|c|}
\hline Vib. State & $\mathrm{C}$ & Exp. & $\begin{array}{c}\text { Exp-Calc } \\
\text { This Work }\end{array}$ & $\begin{array}{c}\text { Morgan [13] } \\
\text { CCSDT(Q) /CBS }\end{array}$ & $\begin{array}{c}\text { Morgan [13] } \\
\text { CCSDT(Q) /CBS+Rel }\end{array}$ \\
\hline$v_{4}$ & $\mathrm{~B}_{1}$ & 1167.256 & 0.27 & -0.8 & -0.95 \\
\hline$v_{6}$ & $\mathrm{~B}_{2}$ & 1249.095 & 0.18 & 0.03 & -0.08 \\
\hline$v_{3}$ & $\mathrm{~A}_{1}$ & 1500.175 & -0.21 & -0.03 & 0.01 \\
\hline$v_{2}$ & $\mathrm{~A}_{1}$ & 1746.009 & -0.44 & -0.32 & -1.48 \\
\hline$v_{1}$ & $\mathrm{~A}_{1}$ & 2782.457 & -0.35 & 1.18 & 0.49 \\
\hline$v_{5}$ & $\mathrm{~B}_{2}$ & 2843.326 & 0.34 & 1.06 & 0.25 \\
\hline MAE & & & 0.29 & 0.57 & 0.54 \\
\hline MAPE(\%) & & & 0.016 & 0.029 & 0.034 \\
\hline RMS & & & 0.31 & 0.73 & 0.75 \\
\hline
\end{tabular}

416

All values are given in $\mathrm{cm}^{-1}$.

417 

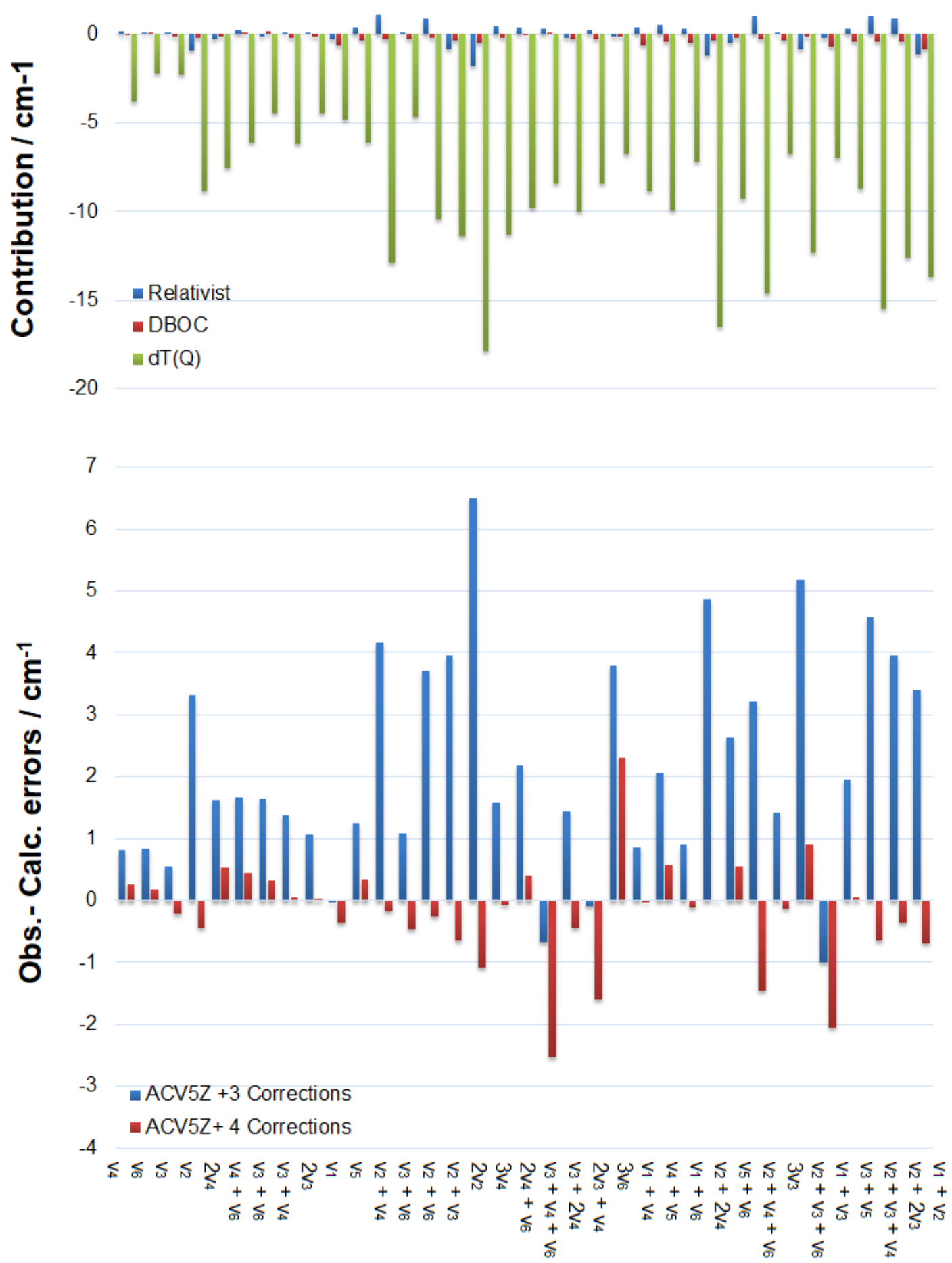

Fig 6. Upper panel(a): contribution of relativistic effects, DBOC, and high-order electron correlations $T(Q)$ to vibrational energies up to $4500 \mathrm{~cm}^{-1}$. Lower panel(b): Experimentalcalculated deviations for vibration levels corresponding to ab initio calculations using PES (ACV5Z+3Corrections) in blue and to PES(ACV5Z+4Corrections) in red. Both vertical scales are given in $\mathrm{cm}^{-1}$. 


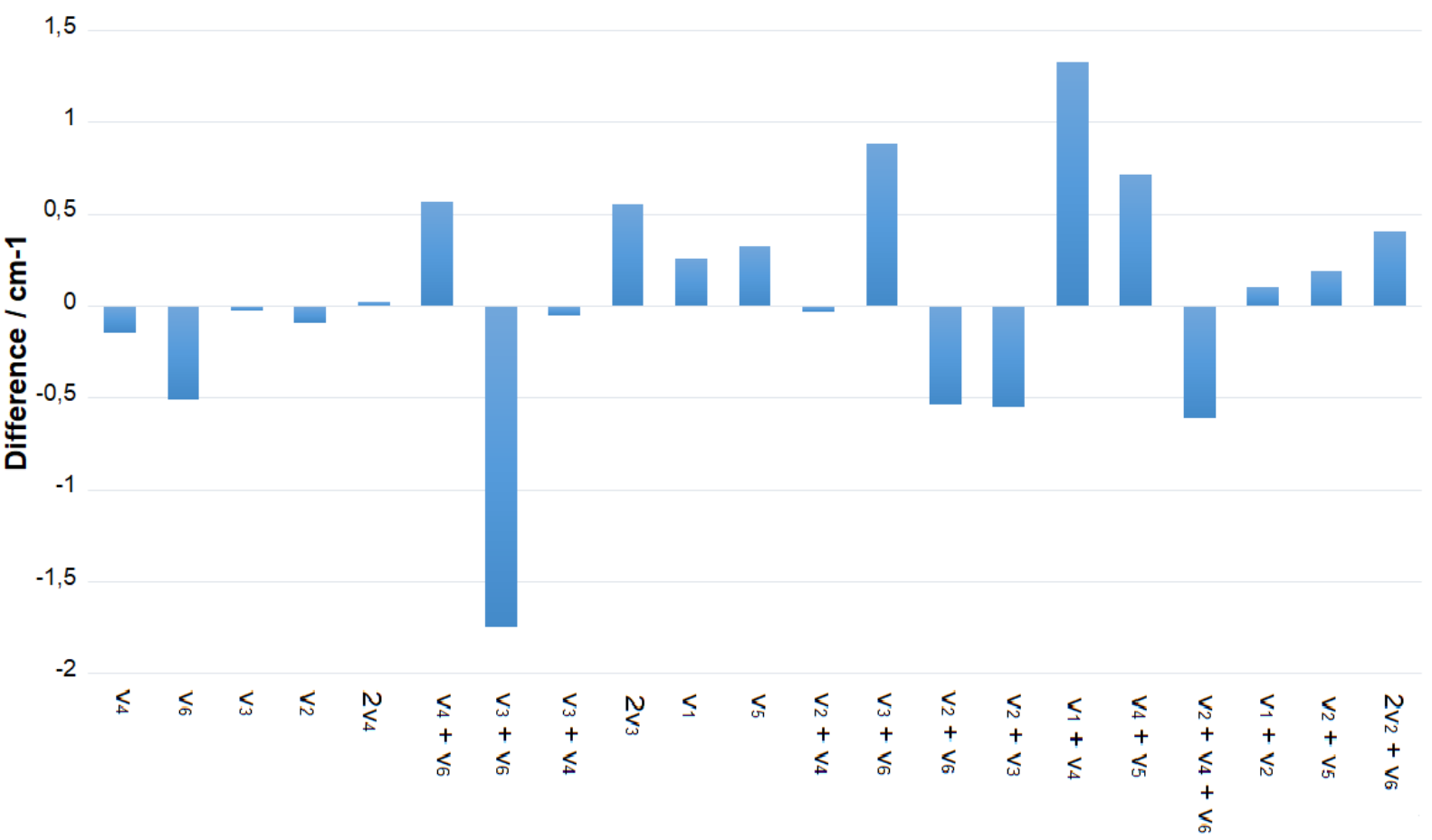

Fig 7. Exp.(HighRes)-Exp.(LowRes), where Exp(HighRes) refers to vibrational levels obtained from high resolution spectra analyses in the infrared range [78] [82] [79], whereas Exp.(LowRes) corresponds to low resolution dispersed fluorescence data reported by Bouwens et al [80]. Vertical scales are given in $\mathrm{cm}^{-1}$.

In order to improve the accuracy of vibrational predictions, we have also produced an empirically optimized PES, by a fitting of 6 harmonic second-order terms of the potential expansion to 20 levels experimentally determined with three decimal digits (reported in Table 5 excepting the level at $4163.289 \mathrm{~cm}^{-1}$ ). With this fine tuning of the six PES parameters, the RMS (exp.-calc.) deviation for 20 accurate levels derived from high-resolution spectra fall down from $0.21 \mathrm{~cm}^{-1}$ to $0.008 \mathrm{~cm}^{-1}$. Figure 8 shows the diagram of differences between the experimental levels and those calculated from our best ab initio PES and from the empirically optimized PES.

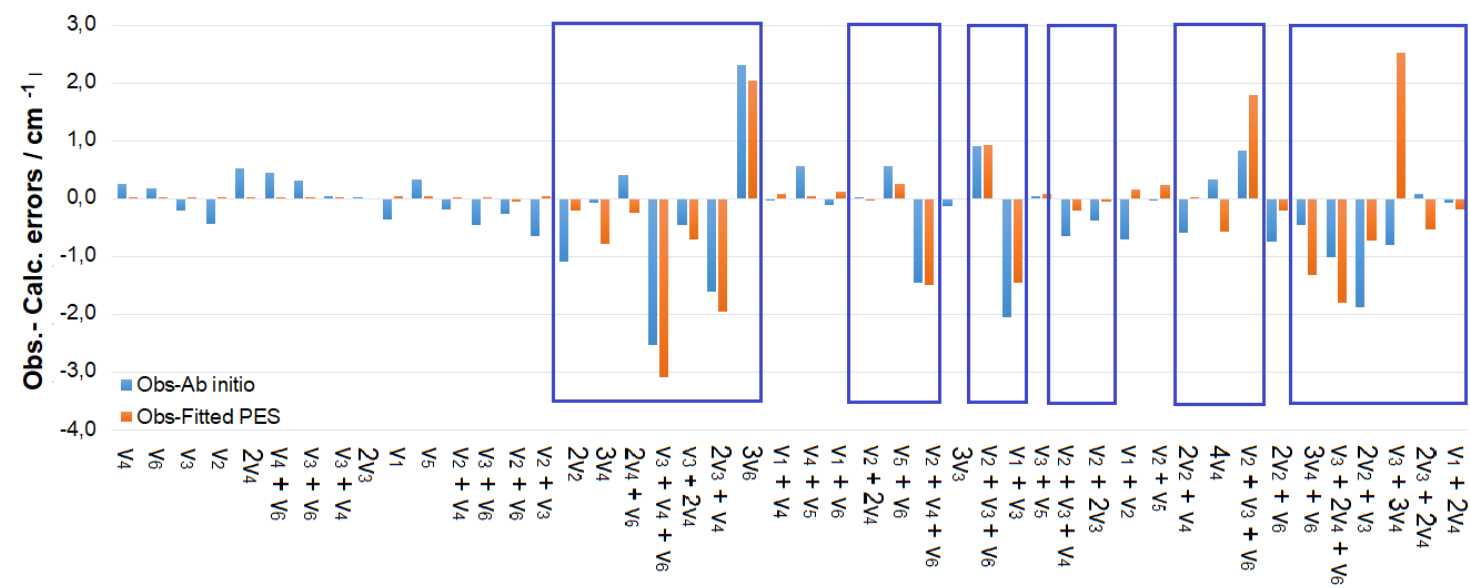

Fig 8. Experimental minus calculated deviations for vibrational levels computed from pure ab 441 initio PES(ACV5Z+4Corr) and from the empirically optimized PES. Vertical scales are given in $\mathrm{cm}^{-1}$. Levels from Bouwens et al. [80] and the suspicious level $v_{2}+v_{4}+v_{6}$ from [79] are 
displayed in rectangles. Only known vibrational levels up to $5100 \mathrm{~cm}^{-1}$ are represented on the

444 horizontal axis.

The experimental values derived from low resolution dispersed fluorescence spectra of Bouwens et al [80], for which the accuracy is considerably lower than for levels obtained from analyses of rotationally resolved infrared spectra using an effective Hamiltonian technique, are shown in the rectangular frame. It is seen that without the levels in the frames, the errors of our ab initio levels are smaller than the discrepancies between the low resolution data of Bouwens et al $[\mathbf{8 0}]$ and the experimental levels obtained in more recent works (see Figure 7). It can thus be concluded that the accuracy of our final ab initio calculations is not worse than that of low resolution experimental levels reported in $[\mathbf{8 0}]$, at least for the bands with doubly and triply excited vibrational quanta. Energy levels up to $7000 \mathrm{~cm}-1$, calculated from our empirically fitted PES in orthogonal coordinates are shown in the first column of Table 8 . These coordinates permit using the KEO, which is considered as "exact" (not requiring series expansion), however the latter term is only meaningful within the framework of the Born-Oppenheimer approximation. In order to calculate energy levels in normal coordinates the energy values generated from the analytical form of the PES in orthogonal coordinates were computed on our $\mathrm{G}^{(\mathrm{R})}$ grid of geometries (see paragraph 2) and then re-fitted in internal coordinates with the torsion function of $(\underline{\tau}-\pi)$. The TENSOR code [83] [84] [85] [86] was then used to calculate the energy levels in normal coordinates using the Watson-Eckart KEO. Most likely, the accuracy of the levels computed from empirically optimized PES for doubly and triply excited quanta is higher than the accuracy of the levels obtained using the pure ab initio PES. However, in case of the bands corresponding to four or more vibrational quanta, the error caused by an empirical correction might be larger than that for levels obtained using the initial ab initio PES, because only 20 low energy experimental levels were included in the fit. Note that four band centers $v_{1}+v_{2}, v_{2}+v_{5}, 2 v_{2}+v_{6}$, and $3 v_{2}$ were not used in fit of the PES, but the corresponding energy levels calculated from empirically optimized PES became noticeably (RMS four times lower) closer to the empirical values. The difference between the two calculations in Table 8 is several times bigger than the initial difference between the corresponding Calc 3 and Calc 4 results for the ab initio V5Z PES (figure 3), although the calculations are converged in both cases. This could occur due to the

473 limited order of the PES expansion and to numerical issues in the procedure of the coordinate transformations on a final grid of points. For this reason, the predicted values of high-energy levels in Table 8 have to be taken with caution. They could be useful in the initial steps of the assignment, if no other, more precise values are available. 
Table 8 . Vibrational levels ( in $\mathrm{cm}^{-1}$ ) of the main formaldehyde isotopologue $\mathrm{H}_{2} \mathrm{CO}$ computed from our empirically optimized PES using two sets of coordinates with two versions of variational calculations.

\begin{tabular}{|c|c|c|c|}
\hline Sym, vib labels* & Calc A $^{\#}$ & Calc B ${ }^{\#}$ & Difference (A-B) \\
\hline B1 000100 & 1167.263 & 1167.247 & 0.016 \\
\hline B2 000001 & 1249.103 & 1249.044 & 0.059 \\
\hline A1 001000 & 1500.140 & 1500.138 & 0.002 \\
\hline A1 010000 & 1746.002 & 1746.005 & -0.003 \\
\hline A1 000200 & 2327.519 & 2327.492 & 0.027 \\
\hline A2 000101 & 2422.989 & 2422.912 & 0.076 \\
\hline A1 000002 & 2494.406 & 2494.293 & 0.113 \\
\hline B1 001100 & 2667.030 & 2667.017 & 0.014 \\
\hline B2 001001 & 2719.222 & 2719.197 & 0.026 \\
\hline A1 100000 & 2782.492 & 2782.499 & -0.008 \\
\hline B2 000010 & 2843.343 & 2843.311 & 0.032 \\
\hline B1 010100 & 2905.969 & 2905.958 & 0.011 \\
\hline A1 002000 & 2998.953 & 2998.941 & 0.012 \\
\hline B2 010001 & 3000.159 & 3000.110 & 0.049 \\
\hline A1 011000 & 3238.402 & 3238.407 & -0.005 \\
\hline A1 020000 & 3471.801 & 3471.806 & -0.005 \\
\hline B1 000300 & 3481.518 & 3481.485 & 0.033 \\
\hline B2 000201 & 3586.906 & 3586.813 & 0.093 \\
\hline B1 000102 & 3676.685 & 3676.568 & 0.117 \\
\hline B2 000003 & 3736.082 & 3735.927 & 0.155 \\
\hline A1 001200 & 3826.023 & 3825.994 & 0.030 \\
\hline A2 001101 & 3888.514 & 3888.480 & 0.034 \\
\hline A1 001002 & 3935.451 & 3935.337 & 0.114 \\
\hline B1 100100 & 3941.539 & 3941.542 & -0.004 \\
\hline A2 000110 & 3996.548 & 3996.503 & 0.045 \\
\hline B2 100001 & 4021.081 & 4021.039 & 0.041 \\
\hline A1 010200 & 4058.360 & 4058.336 & 0.024 \\
\hline A1 000011 & 4082.935 & 4082.874 & 0.061 \\
\hline A2 010101 & 4164.835 & 4164.773 & 0.062 \\
\hline B1 002100 & 4165.153 & 4165.135 & 0.018 \\
\hline B2 002001 & 4192.437 & 4192.407 & 0.030 \\
\hline A1 010002 & 4247.860 & 4247.772 & 0.087 \\
\hline A1 101000 & 4255.327 & 4255.329 & -0.002 \\
\hline B2 001010 & 4335.077 & 4335.028 & 0.049 \\
\hline B1 011100 & 4397.710 & 4397.703 & 0.007 \\
\hline B2 011001 & 4466.891 & 4466.869 & 0.022 \\
\hline A1 003000 & 4495.549 & 4495.533 & 0.016 \\
\hline A1 110000 & 4529.420 & 4529.457 & -0.037 \\
\hline B2 010010 & 4571.525 & 4571.519 & 0.006 \\
\hline B1 020100 & 4624.282 & 4624.281 & 0.001 \\
\hline A1 000400 & 4629.634 & 4629.602 & 0.032 \\
\hline A1 012000 & 4729.001 & 4728.995 & 0.006 \\
\hline B2 020001 & 4734.453 & 4734.419 & 0.035 \\
\hline A2 000301 & 4743.299 & 4743.198 & 0.101 \\
\hline A1 000202 & 4843.901 & 4843.775 & 0.126 \\
\hline A2 000103 & 4928.842 & 4928.695 & 0.147 \\
\hline A1 021000 & 4955.909 & 4955.924 & -0.015 \\
\hline
\end{tabular}




\begin{tabular}{|c|c|c|c|}
\hline A1 000004 & 4974.735 & 4974.536 & 0.199 \\
\hline B1 001300 & 4978.413 & 4978.371 & 0.042 \\
\hline B2 001201 & 5044.315 & 5044.276 & 0.039 \\
\hline A1 100200 & 5092.702 & 5092.730 & -0.027 \\
\hline B1 001102 & 5111.129 & 5111.015 & 0.114 \\
\hline B2 000210 & 5140.810 & 5140.722 & 0.088 \\
\hline B2 000012 & 5151.476 & 5151.302 & 0.175 \\
\hline A1 030000 & 5178.050 & 5178.078 & -0.028 \\
\hline A2 100101 & 5186.406 & 5186.361 & 0.045 \\
\hline B1 010300 & 5205.475 & 5205.445 & 0.029 \\
\hline B1 001102 & 5246.933 & 5246.868 & 0.066 \\
\hline A1 100002 & 5253.556 & 5253.499 & 0.058 \\
\hline B2 010201 & 5312.412 & 5312.323 & 0.089 \\
\hline A1 002200 & 5322.146 & 5322.106 & 0.041 \\
\hline B2 010201 & 5325.537 & 5325.463 & 0.074 \\
\hline A2 002101 & 5359.048 & 5359.007 & 0.041 \\
\hline A1 001011 & 5383.971 & 5383.855 & 0.116 \\
\hline B1 101100 & 5414.636 & 5414.625 & 0.011 \\
\hline B1 010102 & 5419.717 & 5419.635 & 0.082 \\
\hline B2 100010 & 5433.111 & 5433.318 & -0.207 \\
\hline A1 200000 & 5463.113 & 5463.393 & -0.281 \\
\hline A2 001110 & 5490.220 & 5490.169 & 0.051 \\
\hline B2 010003 & 5490.308 & 5490.176 & 0.131 \\
\hline B2 101001 & 5530.358 & 5530.434 & -0.075 \\
\hline A1 011200 & 5544.288 & 5544.261 & 0.026 \\
\hline A1 011200 & 5552.705 & 5552.692 & 0.013 \\
\hline A2 011101 & 5627.353 & 5627.327 & 0.026 \\
\hline A1 000020 & 5651.311 & 5651.317 & -0.007 \\
\hline B1 003100 & 5660.831 & 5660.816 & 0.015 \\
\hline B2 003001 & 5665.587 & 5665.536 & 0.051 \\
\hline B1 110100 & 5681.295 & 5681.319 & -0.025 \\
\hline A1 011002 & 5687.375 & 5687.256 & 0.119 \\
\hline A2 010110 & 5718.950 & 5718.932 & 0.018 \\
\hline A1 102000 & 5729.450 & 5729.448 & 0.002 \\
\hline B2 110001 & 5765.234 & 5765.242 & -0.008 \\
\hline A1 020200 & 5769.551 & 5769.541 & 0.010 \\
\hline B1 000500 & 5772.142 & 5772.133 & 0.009 \\
\hline A1 010011 & 5809.358 & 5809.322 & 0.036 \\
\hline B2 003001 & 5822.554 & 5822.520 & 0.033 \\
\hline B1 012100 & 5887.355 & 5887.344 & 0.011 \\
\hline A2 020101 & 5889.405 & 5889.373 & 0.032 \\
\hline B2 000401 & 5892.767 & 5892.686 & 0.081 \\
\hline B2 012001 & 5935.857 & 5935.799 & 0.057 \\
\hline A1 020002 & 5985.373 & 5985.326 & 0.046 \\
\hline A1 004000 & 5988.654 & 5988.651 & 0.003 \\
\hline A1 111000 & 5997.360 & 5997.369 & -0.009 \\
\hline B1 000302 & 6002.841 & 6002.727 & 0.113 \\
\hline B2 011010 & 6052.566 & 6052.564 & 0.002 \\
\hline B2 000203 & 6097.490 & 6097.335 & 0.155 \\
\hline B1 021100 & 6107.429 & 6107.441 & -0.012 \\
\hline A1 001400 & 6124.578 & 6124.543 & 0.034 \\
\hline B1 000104 & 6180.301 & 6180.124 & 0.177 \\
\hline A2 000310 & 6190.937 & 6190.917 & 0.020 \\
\hline
\end{tabular}




\begin{tabular}{|c|c|c|c|}
\hline B2 021001 & 6196.629 & 6196.618 & 0.011 \\
\hline B2 000005 & 6211.767 & 6211.549 & 0.218 \\
\hline A1 013000 & 6216.906 & 6216.889 & 0.017 \\
\hline B1 100300 & 6236.880 & 6236.997 & -0.117 \\
\hline A1 120000 & 6254.983 & 6255.058 & -0.075 \\
\hline A1 000211 & 6265.909 & 6265.782 & 0.127 \\
\hline B2 020010 & 6275.837 & 6275.861 & -0.024 \\
\hline A2 000310 & 6283.377 & 6283.364 & 0.013 \\
\hline B1 030100 & 6322.849 & 6322.896 & -0.047 \\
\hline A2 000112 & 6335.338 & 6335.170 & 0.168 \\
\hline B2 100201 & 6340.507 & 6340.531 & -0.024 \\
\hline A1 010400 & 6346.545 & 6346.522 & 0.022 \\
\hline A1 000013 & 6360.567 & 6360.303 & 0.264 \\
\hline A1 001202 & 6401.440 & 6401.405 & 0.036 \\
\hline B1 100102 & 6426.649 & 6426.645 & 0.004 \\
\hline A1 022000 & 6437.667 & 6437.681 & -0.014 \\
\hline B2 030001 & 6451.851 & 6451.859 & -0.008 \\
\hline A2 010301 & 6466.652 & 6466.599 & 0.053 \\
\hline B1 002300 & 6472.643 & 6472.596 & 0.047 \\
\hline B2 100003 & 6476.996 & 6476.944 & 0.052 \\
\hline A2 001103 & 6495.501 & 6495.445 & 0.056 \\
\hline B2 001210 & 6510.481 & 6510.441 & 0.039 \\
\hline A1 001004 & 6546.991 & 6546.894 & 0.097 \\
\hline B1 001111 & 6553.062 & 6553.068 & -0.006 \\
\hline A1 101200 & 6564.842 & 6564.913 & -0.071 \\
\hline B2 001012 & 6578.550 & 6578.313 & 0.237 \\
\hline A1 010202 & 6580.280 & 6580.214 & 0.066 \\
\hline A2 100110 & 6584.443 & 6584.991 & -0.548 \\
\hline B1 200100 & 6612.308 & 6612.932 & -0.624 \\
\hline A1 100011 & 6629.339 & 6629.655 & -0.317 \\
\hline B2 002201 & 6636.922 & 6636.909 & 0.013 \\
\hline A1 031000 & 6653.251 & 6653.314 & -0.062 \\
\hline A2 010103 & 6670.621 & 6670.512 & 0.109 \\
\hline A2 101101 & 6683.934 & 6684.163 & -0.229 \\
\hline B2 200001 & 6684.586 & 6685.095 & -0.509 \\
\hline B1 011300 & 6693.205 & 6693.178 & 0.027 \\
\hline B1 002102 & 6708.592 & 6708.624 & -0.032 \\
\hline A1 010004 & 6727.367 & 6727.179 & 0.188 \\
\hline B2 002003 & 6758.622 & 6758.625 & -0.003 \\
\hline A1 101002 & 6766.827 & 6767.112 & -0.285 \\
\hline B2 011201 & 6778.065 & 6778.064 & 0.001 \\
\hline B1 000120 & 6795.388 & 6795.461 & -0.074 \\
\hline A1 003200 & 6813.391 & 6813.333 & 0.057 \\
\hline A1 110200 & 6825.366 & 6825.462 & -0.096 \\
\hline A2 002110 & 6829.984 & 6829.955 & 0.030 \\
\hline A1 002011 & 6838.098 & 6838.072 & 0.026 \\
\hline B1 011102 & 6851.401 & 6851.322 & 0.079 \\
\hline B2 010210 & 6856.265 & 6856.337 & -0.071 \\
\hline B2 101010 & 6864.634 & 6865.029 & -0.395 \\
\hline A1 040000 & 6865.517 & 6865.643 & -0.126 \\
\hline B1 102100 & 6888.866 & 6888.915 & -0.049 \\
\hline B2 011003 & 6894.266 & 6894.350 & -0.083 \\
\hline B2 010012 & 6904.180 & 6903.974 & 0.206 \\
\hline
\end{tabular}




\begin{tabular}{|c|c|c|c|}
\hline A1 000600 & 6909.239 & 6909.337 & -0.098 \\
\hline B1 020300 & 6909.809 & 6909.812 & -0.003 \\
\hline A1 201000 & 6914.801 & 6915.485 & -0.684 \\
\hline A2 110101 & 6922.631 & 6922.694 & -0.063 \\
\hline B1 011102 & 6966.384 & 6966.388 & -0.003 \\
\hline A2 003101 & 6978.675 & 6978.675 & 0.000 \\
\hline A1 110002 & 6990.034 & 6990.107 & -0.074 \\
\hline
\end{tabular}

*) Vibrational state labels include $C_{2 v}$ symmetry types and normal mode vibrational assignment.

${ }^{\#)}$ Calc A. corresponds to direct calculations using our empirically optimized PES in orthogonal coordinates (I) and the torsion functions $\cos (\tau / 2)$ with the exact kinetic energy operator in polyspherical coordinates.

${ }^{\# \#)}$ Calc B. corresponds to calculations using our empirically optimized PES refitted in normal coordinates on a geometrical grid with the Watson-Eckart kinetic energy operator.

\section{Deuterated $D_{2} C O$ isotopologue}

The vibrational levels of $\mathrm{D}_{2} \mathrm{CO}$ given in Table 9 were calculated, using nuclear masses, from two potential surfaces. The calculation $(\underline{\mathrm{CalcD}} 2 \underline{\mathrm{CO}} 1)$ corresponds to our best ab initio PES(ACV5Z+4Corr) and $\left(\underline{\mathrm{CalcD}}_{2} \underline{\mathrm{CO}} 2\right)$ corresponds to the empirically optimized PES fitted to twenty vibrational levels of the main formaldehyde isotopologue as described in the previous section. Experimental levels were taken from refs [82] (the first 3 levels), [87] (v2), and [88] (the remaining 8 levels). Note that ref $[\mathbf{8 8}]$ reports both the vibrational levels obtained from experimental transitions from the ground state to the upper level $\mathrm{J}=0$ (see Table 3 in ref [88]), as well as the spectroscopic parameter of the band center derived with the least squares technique. The spectroscopic parameter of the band center and vibrational level in the same units (cm-1) should have closely lying values in the absence of anharmonic Fermi-type resonances, but could considerably differ however, in the case of a strong Fermi coupling. Table 10 of ref [13] erroneously gives the spectroscopic parameter $2054.694 \mathrm{~cm}^{-1}$ instead of the experimental vibrational level $2060.91963 \mathrm{~cm}^{-1}$ for $v_{1}$. The experimental vibrational level $\mathrm{v}_{1}$ is considerably closer to the calculated value of ref [13]. In Table 9, we compare the experimental and calculated levels. Note that all calculated levels, except for $2 v_{4}$, are close to experimental ones. The choice of various representations of potentials, coordinates, and the size of the vibrational basis do not have any considerable effect on the calculated energy levels given in Table 9. The $\mathrm{D}_{2} \mathrm{CO}$ isotopologue is heavier than $\mathrm{H}_{2} \mathrm{CO}$, and the $\mathrm{DBOC}$ correction for $\mathrm{D}_{2} \mathrm{CO}$ is expected to be less important than this correction for $\mathrm{H}_{2} \mathrm{CO}$. In case of D2CO $\left(\mathrm{CalcD}_{2} \mathrm{CO} 1\right)$, the DBOC was not recalculated. To estimate the contribution of DBOC, we presented it in the form of an expansion in a second-order Taylor series. Comparison of parameters of the DBOC of the two isotopologues $\mathrm{D} 2 \mathrm{CO} / \mathrm{H} 2 \mathrm{CO}$ shows that the contribution of DBOC_D2CO is 0.6 *DBOC $\mathrm{H} 2 \mathrm{CO}$. Since the contribution is weak, the -0.4 contribution for the main isotopologue (see column DBOC of Table 5) could be added to the calculated D2CO levels (CalcD 2 CO 1). In 

closer to the empirical values.

Since a considerable number of transitions in the $2 v_{4}$ band have been assigned and accurate combination differences have been derived in ref [88], an outlier in the $2 v_{4}$ band center printed in

518 Table 3 of ref [88] could likely be interpreted as a misprint. On the other hand, the authors of ref 519 [88] have stated (the second sentence of the fourth paragraph): "One can clearly see the three 520 strong fundamental bands $v_{2}, v_{1}$, and $v_{5}$ with the centers located near $1701.6 \mathrm{~cm}^{-1}, 2060.9 \mathrm{~cm}^{-1}$, 521 and $2162.9 \mathrm{~cm}-1$, respectively. The weak bands $2 v_{4}, 1867.8 \mathrm{~cm}^{-1}, v_{4}+v_{6}, 1930.0 \mathrm{~cm}^{-1}$, and $2 v_{6}$, $5221974.3 \mathrm{~cm}^{-1}$, can also be recognized very clearly in spite of their weakness". These band centers 523 are close (within $0.1 \mathrm{~cm}^{-1}$ ) to reported experimental vibrational levels of ref [88], except for the 524 experimental vibrational level $2 v_{4}$, which was shifted by $2 \mathrm{~cm}^{-1}$ with respect to the band center 525 cited above. Note, that the center of the $2 v_{4}$ band reported in ref [88] $\left(1867.8 \mathrm{~cm}^{-1}\right)$ is very close 526 to our calculated vibrational level of $2 v_{4}\left(1867.6 \mathrm{~cm}^{-1}\right)$. Figure 7 of ref $[\mathbf{8 8}]$ indicates the 527 presence of a Fermi resonance between the $2 v_{4}$ and $v_{1}$ bands. However, Table 6 of the same 528 paper $[\mathbf{8 8}]$ missed the parameter of this resonance.

529 The last row of Table 9 gives the RMS values of differences between experimental and 530 calculated levels. The empirically optimized PES provides the best description of the energy 531 levels, except for the doubted $2 v_{4}$ value.

532 Table 9. Vibrational levels for the $D_{2} C O$ isotopologue up to $3000 \mathrm{~cm}^{-1}$.

\begin{tabular}{|c|c|c|c|c|c|c|}
\hline Vib. State & $\mathrm{C}$ & Exp. levels *) & 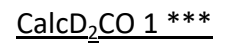 & Exp.-CalcD 2 & $\underline{\text { CalcD }}_{2}{ }_{2} \underline{2_{2}^{* * *}}$ & Exp.-CalcD 2 \\
\hline$v_{4}$ & $\mathrm{~B}_{1}$ & 938.03549 & 937.936 & 0.099 & 937.711 & 0.324 \\
\hline$v_{6}$ & $\mathrm{~B}_{2}$ & 989.25028 & 989.174 & 0.076 & 989.053 & 0.197 \\
\hline$v_{3}$ & $\mathrm{~A}_{1}$ & 1100.44254 & 1100.403 & 0.039 & 1100.536 & -0.093 \\
\hline$v_{2}$ & $\mathrm{~A}_{1}$ & 1701.619103 & 1701.166 & 0.453 & 1701.631 & -0.012 \\
\hline $2 v_{4}$ & $\mathrm{~A}_{1}$ & 1865.84444 & 1867.602 & -1.757 & 1867.21 & -1.365 \\
\hline$v_{4}+v_{6}$ & $\mathrm{~A}_{2}$ & 1930.02676 & 1929.852 & 0.174 & 1929.524 & 0.502 \\
\hline $2 v_{6}$ & $\mathrm{~A}_{1}$ & 1974.32541 & 1974.232 & 0.092 & 1973.993 & 0.332 \\
\hline$v_{3}+v_{4}$ & $\mathrm{~B}_{1}$ & 2038.90981 & 2038.801 & 0.108 & 2038.708 & 0.202 \\
\hline$v_{1}$ & $\mathrm{~A}_{1}$ & 2060.91963 & 2060.643 & 0.275 & 2060.852 & 0.067 \\
\hline$v_{3}+v_{6}$ & $\mathrm{~B}_{2}$ & 2072.66611 & 2072.585 & 0.080 & 2072.509 & 0.157 \\
\hline$v_{5}$ & $\mathrm{~B}_{2}$ & 2162.92330 & 2162.509 & 0.414 & 2162.118 & 0.805 \\
\hline $2 v_{3}$ & $\mathrm{~A}_{1}$ & 2201.73284 & 2201.727 & 0.005 & 2201.98 & -0.247 \\
\hline$v_{2}+v_{4}$ & $\mathrm{~B}_{1}$ & & 2633.045 & & 2633.258 & \\
\hline$v_{2}+v_{6}$ & $\mathrm{~B}_{2}$ & & 2685.004 & & 2685.288 & \\
\hline$v_{2}+v_{3}$ & $\mathrm{~A}_{1}$ & & 2790.027 & & 2789.501 & \\
\hline $\begin{array}{l}\text { RMS deviation } \\
\left.\text { RMS ( - level } 2 v_{4}\right)\end{array}$ & & & & $\begin{array}{l}0.55 \\
0.21\end{array}$ & & $\begin{array}{l}0.51 \\
0.34\end{array}$ \\
\hline
\end{tabular}

*) Experimental levels from refs [82] [87] [88] in $\mathrm{cm}^{-1}$.

$534 * *$ ) RMS deviation without the suspicious level $2 v_{4}$.

$535 * * *$ ) See text in the beginning of the paragraph 


\section{Conclusion}

The recent thorough study of the quartic force field of the formaldehyde molecule by Morgan et al [13] obtained at 183 nuclear geometries near the equilibrium configuration has shown the importance of high-order dynamic electron correlation by including triple and quadruple excitations in the coupled-cluster hierarchy via the CCSDT(Q) method. This has permitted improving fundamental band origins with respect to the standard $\operatorname{CCSD}(\mathrm{T})$ approach with an RMS deviation of $0.52 \mathrm{~cm}^{-1}$ [13]. In this work, we confirm these conclusions using the algorithm of the construction of the full six-dimensional PES at an extended set of geometries. To this end, we used successive grids adapted to the level of the theory that was previously applied for the methane molecule [12]. These methods, including also relativistic and adiabatic DBOC corrections, allowed us to approach the spectroscopic accuracy in ab initio calculations for a larger range of vibrational energies. For the first time - in case of a four-atom molecule with $\underline{16}$ electrons like $\mathrm{H} 2 \mathrm{CO}$ - it was possible to obtain the (ab initio - experimental) RMS deviation of $0.4 \mathrm{~cm}^{-1}$ including 33 experimental vibrational band origins up to three vibrational quanta (Table $6)$.

First-principle calculations provide an independent insight into uncertainties of available experimental data, particularly for those which had been deduced from low-resolution dispersed fluorescence spectra [80]. A detailed comparison of various sources in Table 7 and Figures 6-8 suggests that many experimental levels could be less accurate than the present ab initio calculations. By excluding five suspicious band origins corresponding to large experimental uncertainties, the RMS deviation of our ab initio PES for vibrational levels up to $3700 \mathrm{~cm}-1$ (Table 6) drops down to $0.2 \mathrm{~cm}^{-1}$. A similar accuracy is obtained for vibrational levels of the deuterated isotopologue D2CO (Table 9), confirming the reliability of the ab initio PES.

We have also produced an empirically optimized PES by adjusting six second order parameters to twenty experimental vibrational levels derived from high-resolution spectra with the (obs-calc) RMS deviation of $0.008 \mathrm{~cm}^{-1}$. Computed from this PES 160 band origins of H2CO up to $7000 \mathrm{~cm}^{-1}$ are given in Table 8 . Both the ab initio and the empirically optimized PES are provided in the Supplementary Materials as a $\mathrm{C}++$ code.

For a further investigation of the corresponding accuracy issues, the ab initio PES can be used to derive effective spectroscopic models by the contact transformation method as it was done in the case of methane [89] [90] [91]. This will permit to accurately compute physically meaningful values of the resonance coupling parameters from ab initio surface for advanced analyses of high-resolution spectra. 


\section{SUPPLEMENTARY MATERIAL}

573 See supplementary material for the ab initio and the empirically optimized PES in orthogonal

574 coordinates.

\section{ACKNOWLEDGMENTS}

The support of RFBR grant (Grant No. 19-03-00581) is acknowledged. V.T. acknowledges the support from Academic D.Mendeleev program of Tomsk State University and M.R. form LEFE CHAT 579 CNRS program.

\section{References}

[1] Barbe A., Marche' P., Secroun C., Jouve P., Measurements of tropospheric and stratospheric H2CO by an infrared high resolution technique. J. Geophys. Res. Lett. 1979;6: 463-465.

[2] Khare P., Kumar N., Kumari K.M., Srivastave S.S., Atmospheric formic and acetic acids: An overview. Rev. Geophys. 1999;37: 227.

[3] Kakabokas P., Carlier P., Fresnet P., Mouvierg G., Toupance G., Field studies of aldehyde chemistry in the Paris area. Atmos. Environ. 1988;22: 147-155.

[4] Zhu L., Abad G.G., Nowlan C.R., Miller C.C., Chance K, Ape E.C., DiGangi J.P., Fried A., Hanisco T.F., Hornbrook R.S., Hu L., Validation of satellite formaldehyde (HCHO) retrievals using observations from 12 aircraft campaigns Atmos. Chem. Phys. 2020;20: 12329-12345.

[5] Steck T., Glatthor N., Von Clarmann T., Fischer H., Flaud J.M., Funke B., Grabowski U., Höpfner M., Retrieval of global upper tropospheric and stratospheric formaldehyde $(\mathrm{H} 2 \mathrm{CO})$ distributions from high-resolution MIPAS-Envisat spectra Atmospheric Chemistry and Physics 2008;8: 463-470.

[6] Dufour G., Szopa S., Barkley M.P., Boone C.D., Perrin A., Palmer P.I., Bernath P.F., Global uppertropospheric formaldehyde: Seasonal cycles observed by the ACE-FTS satellite instrument Atmospheric Chemistry and Physics 2009;9: 3893-3910.

[7] Rey M., Nikitin A.V., Tyuterev V.G., Accurate Theoretical Methane Line Lists in the Infrared up to $3000 \mathrm{~K}$ and Quasi-continuum Absorption/Emission Modeling for Astrophysical Applications Astrophysical journal 2017;847: 105.

[8] Rey M., Nikitin A.V., Campargue A., Kassi S., Mondelain D., Tyuterev VI.G., Ab initio variational predictions for understanding highly congested spectra: Rovibrational assignment of 108 new methane sub-bands in the icosad range (6280-7800 cm-1) Phys. Chem.Chem. Phys. 2016;16: 176189.

[9] Wong A, Bernath P.F., Rey M., Nikitin A.V., Tyuterev V.G., Atlas of Experimental and Theoretical High-temperature Methane Cross Sections from T=295 to 1000K in the Near-infrared The 
Astrophysical Journal Supplement Series 2019;240: 4.

[10] Nikitin A.V., Ivanova Y.A., Rey M., Tashkun S.A., Toon G.C., Sung, K., Tyuterev VI.G., Analysis of PH3 spectra in the Octad range 2733-3660 cm-1 J. Quant. Spectrosc. Radiat. Transf. 2017;203: 472-479.

[11] Rey M., Chizhmakova I.S., Nikitin A.V., Tyuterev VI.G., Understanding global infrared opacity and hot bands of greenhouse molecules with low vibrational modes from first-principles calculations: The case of CF4 Phys. Chem.Chem. Phys. 2018;20, no. 32: 21008-21033.

[12] Nikitin A.V., Rey M., Tyuterev VI.G., First fully ab initio potential energy surface of methane with a spectroscopic accuracy J. Chem. Phys. 2016;145: 114309.

[13] Morgan W.J., Matthews D.A., Ringholm M., Agarwal J., Gong J.Z., Ruud K., Allen W.D., Stanton J.F., Schaefer H.F., Geometric Energy Derivatives at the Complete Basis Set Limit: Application to the Equilibrium Structure and Molecular Force Field of Formaldehyde J. Chem. Theory Comput. 2018;14: 1333-1350.

[14] Yachmenev A., Yurchenko S.N., Jensen P., Thiel W., A new "spectroscopic" potential energy surface for formaldehyde in its ground electronic state J. Chem. Phys. 2011;134: 244307-12.

[15] Burleigh D.C., McCoy A.B., Sibert E.L. III, An accurate quartic force field for formaldehyde J. Chem. Phys 1996;104: 480.

[16] Martin, J. M. L.; Lee, T. J., An accurate ab initio Ouartic Force Field for Formaldehyde and its Isotopomers J. Molec. Spectrosc. 1993;160: 105-116.

[17] Burleigh D.C. , Sibert E.L., A random matrix approach to rotation-vibration mixing in $\mathrm{H} 2 \mathrm{CO}$ and D2CO J. Chem. Phys. 1993;98: 8419-8431.

[18] Mladenovic M., Discrete variable approaches to tetratomic molecules Part II: application to $\mathrm{H} 2 \mathrm{O} 2$ and H2CO Spectrochimica Acta Part A 2002;58: 809-824.

[19] Yagi K., Oyanagi C., Taketsugu T., Hirao K., Ab initio potential energy surface for vibrational state calculations of H2CO J. Chem. Phys. 2003;118: 1653-1660.

[20] Al-Refaie A. F., Yachmenev A., Tennyson J., Yurchenko S. N., "ExoMol line lists - VIII. A variationally computed line list for hot formaldehyde," MNRAS, vol. 448, pp. 1704-1714, 2015.

[21] Partridge H. , Schwenke D.W., The determination of an accurate isotope dependent potential energy surface for water from extensive ab initio calculations and experimental data J. Chem. Phys. 1997;106: 4618-4639.

[22] Polyansky O.L., Zobov N.F., Viti S., Tennyson J, Bernath P.F., Wallace L., Water on the sun:line assignments based on variational calculations. Science 1997;277, no. 5324: 346-8.

[23] Tennyson J., Barletta P., Kostin M.A., Polyansky O.L., Zobov N.F., Ab initio rotation-vibration energy levels of triatomics to spectroscopic accuracy Spectrochimica Acta A 2002;58, no. 4: 663-672. 
[24] Polyansky O., Ovsyannikov R., Kyuberis A., Lodi L., Tennyson J., Zobov N., Calculation of RotationVibration Energy Levels of the Water Molecule with Near-Experimental Accuracy Based on an ab Initio Potential Energy Surface. J. Phys. Chem. A 2013;117, no. 39: 9633-9643.

[25] Huang X., Schwenke D.W., Tashkun S.A., al. et, An isotopic-independent highly accurate potential energy surface for $\mathrm{CO} 2$ isotopologues and an initial (C16O2)-C-12 infrared line list J. Chem. Phys. 2012;136: 124311.

[26] Huang X., Freedman R.S., Tashkun S.A., Schwenke D.W., Lee T.J., Semiempirical 12C16O2 IR line lists for simulations up to $1500 \mathrm{~K}$ and $20000 \mathrm{~cm}-1$. J.Quant.Spectrosc. Radiat.Transf. 2013;139: 134-46.

[27] Yurchenko S.N., Barber R.J., Yachmenev A., Thiel W., Jensen P., Tennyson J., A Variationally Computed T = 300 K Line List for NH3 J. Phys. Chem. A 2009;113, no. 43: 11845-11855.

[28] Cours T., Rosmus P., Tyuterev V.G., Ab initio dipole moment functions of $\mathrm{H} 232 \mathrm{~S}$ and intensity anomalies in rovibrational spectra. J. Chem. Phys. 2002;117, no. 11: 5192-5208.

[29] Azzam A.A., Lodi L., Yurchenko S.N., Tennyson J., The dipole moment surface for hydrogen sulfide H2S J.Quant.Spectrosc.Radiat.Transf. 2015;161: 41-49.

[30] Huang X.C., Schwenke D.W., T.J. Lee, Highly accurate potential energy surface, dipole moment surface, rovibrational energy levels, and infrared line list for (SO2)-S-32-0-16 up to $8000 \mathrm{~cm}-1 \mathrm{~J}$. Chem. Phys. 2014;140, no. 11: 114311.

[31] Tyuterev VI.G., Kochanov R.V., Tashkun S.A., Holka F., Szalay P.G., New analytical model for the ozone electronic ground state potential surface and accurate ab initio vibrational predictions at high energy range J. Chem. Phys. 2013;139: 134307.

[32] Barbe A., Mikhailenko S., Starikova E., al. et, Ozone spectroscopy in the electronic ground state: High-resolution spectra analyses and update of line parameters J. Quant. Spectrosc. Radiat. Transf. 2013;130: 172-190.

[33] Tyuterev VI.G., Kochanov R.V., Campargue A., Kassi S., Mondelain D., Barbe A., Does the "reef structure" at the ozone transition state towards the dissociation exist? New insight from calculations and ultrasensitive spectroscopy experiments Phys.Rev.Lett 2014;113: 143002.

[34] Tyuterev V.G., Barbe A., Jacquemart D., Janssen C., Mikhailenko S.N., Starikova E.N., Ab initio predictions and laboratory validation for consistent ozone intensities in the $\mathrm{MW}, 10$ and $5 \mu \mathrm{m}$ ranges J. Chem. Phys. 2019;150, no. 18: 184303.

[35] Dawes R., Lolur P., Ma J., Guo H., Highly accurate ozone formation potential and implications for kinetics. J. Chem. Phys. 2011;135: 081102.

[36] Kokoouline V., Lapierre D., Alijah A., Tyuterev VI.G., Localized and delocalized bound states of the main isotopologue 4803 and of 180 -enriched 5003 isotopomers of the ozone molecule near the dissociation threshold. Phys. Chem. Chem. Phys. 2020;22: 15885 - 15899.

[37] Yuen C.H., Lapierre D., Gatti F., Kokoouline V., Tyuterev V.G., The Role of Ozone Vibrational Resonances in the Isotope Exchange Reaction 160160+180 $\rightarrow$ 180160+160: The Time-Dependent 
Picture J. Chem. Phys. 2019;123, no. 36: 7733-7743.

[38] Huang X., Schwenke D.W., Lee T.J., Rovibrational spectra of ammonia. I. Unprecedented accuracy of a potential energy surface used with nonadiabatic corrections J. Chem. Phys. 2011;134, no. 4: 044320 .

[39] Yurchenko S.N., Carvajal M., Thiel W, Jensen P., Ab initio dipole moment and theoretical rovibrational intensities in the electronic ground state of PH3 J. Molec. Spectrosc. 2006;239: 71-87.

[40] Sousa-Silva C., Yurchenko S.N., Tennyson J., A computed room temperature line list for phosphine $J$. Molec. Spectrosc. 2013;288: 28-36.

[41] Nikitin A.V., Rey M., Tyuterev VI.G, High order dipole moment surfaces of PH3 and ab initio intensity predictions in the Octad range J. Molec. Spectrosc 2014;305: 40-47.

[42] Owens A., Yurchenko S.N., Yachmenev A., Tennyson J., Thiel W., Accurate ab initio vibrational energies of methyl chloride J. Chem. Phys. 2015;142: 244306.

[43] Owens A., Yurchenko S.N., Yachmenev A., Thiel W., A global potential energy surface and dipole moment surface for silane J. Chem. Phys. 2015;143: 244317.

[44] Delahaye T., Nikitin A., Rey M., Szalay P., Tyuterev VI.G., A new accurate ground-state potential energy surface of ethylene and predictions for rotational and vibrational energy levels J. Chem. Phys. 2014;141: 104301.

[45] Delahaye T., Nikitin A.V., Rey M., Szalay P.G., Tyuterev V.G., Accurate 12D dipole moment surfaces of ethylene Chem. Phys. Letters 2015;639: 275-282.

[46] Li J., Carter S., Bowman J.M., Dawes R., Xie D., Guo H., High-Level, First-Principles, Full-Dimensional Quantum Calculation of the Ro-vibrational Spectrum of the Simplest Criegee Intermediate $(\mathrm{CH} 2 \mathrm{OO})$ J. Phys. Chem. Lett. 2014;5, no. 13: 2364-2369.

[47] Nikitin A.V., Rey M., Chizhmakova I.S., Tyuterev VI.G., First Full-Dimensional Potential Energy and Dipole Moment Surfaces of SF6 J. Phys. Chem. A 2020;124, no. 35: 7014-7023.

[48] Polyansky O., Kyberis A., Zobov N., Tennyson J., Yurchenko S., Lodi L., ExoMol molecular line lists XXX: a complete high-accuracy line list for water MNRAS 2018;480, no. 2: 2597-2608.

[49] Lukka T.J., A simple method for the derivation of exact quantum-mechanical vibration-rotation Hamiltonians in terms of internal coordinates J. Chem. Phys. 1995;102: 3945.

[50] Colwell S.M. , Handy N.C., The derivation of vibration-rotation kinetic energy operators in internal coordinates. II Molec. Phys. 1997;92: 317.

[51] Bramley M.J. , Carrington T.Jr., A general discrete variable method to calculate vibrational energy levels of three- and four-atom molecules J. Chem. Phys. 1993;99: 8519-8541.

[52] Poulin N.M., Bramley M.J., Jr. CarringtonT., Kjaergaard H.G., Henry B.R., Calculation of vibrational $(J=0)$ excitation energies and band intensities of formaldehyde using the recursive residue 
generation method. J. Chem. Phys. 1996;104: 7807-7820.

[53] Makarewicz J. , Skalozub A., Rovibrational Molecular Hamiltonian in Mixed Bond-Angle and Umbrella-Like Coordinates J. Phys. Chem. A. 2007;111: 7860-7869.

[54] Nikitin A.V., Holka F., Tyuterev VI.G., Fremont J., Vibration energy levels of the PH3, PH2D, and PHD2 molecules calculated from high order potential energy surface J. Chem. Phys 2009;131: 244312.

[55] Nikitin A.V., Rey M., Tyuterev VI.G., Rotational and vibrational energy levels of methane calculated from a new potential energy surface Chem. Phys. Lett. 2011;501: 179-186.

[56] Werner H.-J., Knowles P.J., Knizia G., Manby F.R., al. et, Molpro: a general purpose quantum chemistry program package WIREs Comput Mol Sci 2012;2: 242--253.

[57] Werner H.- J., Knowles P.J, Lindh R., al. et., MOLPRO, version 2019.1, a package of ab initio programs; http://www.molpro.net.

[58] Schwenke D.W. , Partridge H., Vibrational energy levels for $\mathrm{CH} 4$ from an ab initio potential Spectrochim. Acta A 2001;57: 887.

[59] Brünken S., Müller H.S. P., Lewen F., High accuracy measurements on the ground state rotational spectrum of formaldehyde (H2CO) up to 2 THz. Phys. Chem. Chem. Phys. 2003;5: 1515-1518.

[60] Müller H.S. P. , Lewen F., Submillimeter spectroscopy of $\mathrm{H} 2 \mathrm{C} 17 \mathrm{O}$ and a revisit of the rotational spectra of H2C18O and H2C16O. J. Molec. Spectrosc. 2017;331: 28-33.

[61] Muller H.S.P., Winnewisser G., Demaison J., Perrin A., Valentin A., The Ground State Spectroscopic Constants of Formaldehyde J. Molec. Spectrosc. 2000;200: 143-144.

[62] M. Born , Huang K., Dynamical theory of crystal lattices, Oxford at the Clarendon Press. Oxford at the Clarendon Press: Clarendon Press, 1954.

[63] Handy N.C., Yamaguchi Y., Schaefer H.F., The diagonal correction to the Born-Oppenheimer approximation: Its effect on the singlet-triplet splitting of $\mathrm{CH} 2$ and other molecular effects. J. Chem. Phys. 1986;84: 4481-4484.

[64] Cencek W. , Kutzelnigg W., Accurate adiabatic correction for the hydrogen molecule using the BornHandy formula. Chem. Phys. Lett. 1997;266: 383-387.

[65] Gauss J., Tajti A., Kállay M., Stanton J.F., Szalay P.G., Analytic calculation of the diagonal BornOppenheimer correction within configuration-interaction and coupled-cluster theory. J. Chem. Phys. 2006;125: 144111.

[66] Tajti A., Szalay P.G., Gauss J., Perturbative treatement of the electron-correlation contribution to the diagonal Born-Oppenheimer correction. J. Chem. Phys. 2007;127: 014102.

[67] Szalay P.G., Holka F., Fremont J., Rey M, Peterson K.A., Tyuterev VI.G., Are ab initio quantum chemistry methods able to predict vibrational states up to the dissotiation limit for multi-electron 
molecules close to spectroscopic accuracy? Phys. Chem. Chem. Phys. 2011;13: 3654-3659.

[68] Holka F., Szalay P.G., Fremont J., Rey M., Peterson K.A., Tyuterev VI.G., Accurate ab initio determination of the adiabatic potential energy function and the Born-Oppenheimer breakdown corrections for the electronic ground state of LiH isotopologues. J. Chem. Phys. 2011;134: 094306.

[69] Noga J. , Bartlett R.J., The full CCSDT model for molecular electronic structure. J. Chem. Phys. 1987;86: 7041.

[70] Oliphant N. , Adamowicz L., Coupled-cluster method truncated at quadruples. J. Chem. Phys. 1991;95: 6645

[71] Kucharski S.A. , Bartlett R.J., The coupled-cluster single, double, triple, and quadruple excitation method. J. Chem. Phys. 1992;97: 4282.

[72] Bomble Y.J., Stanton J.F., Kállay M., Gauss J., Coupled cluster methods including noniterative corrections for quadruple excitations. J. Chem. Phys. 2005;123: 054101.

[73] Matthews D.A. , Stanton J.F., Non-orthogonal spin-adaptation of coupled cluster methods: A new implementation of methods including quadruple excitations J. Chem. Phys. 2015;142: 064108.

[74] Matthews D.A. , Stanton J.F., Accelerating the convergence of higher-order coupled cluster methods. J. Chem. Phys. 2015, 143, 204103 2015;143: 204103.

[75] Owens A., Yurchenko S.N., Yachmenev A., Tennyson J., Thiel W., A highly accurate ab initio potential energy surface for methane J. Chem. Phys. 2016;145: 104305.

[76] Stanton J.F., Gauss J., Harding M.E., Szalay P.G., al. et. CFour program http://www.cfour.de.

[77] Perez R., Brown J.M., Utkin Y., Han J., Curl R.F., Observation of hot bands in the infrared spectrum of H2CO J. Molec. Spectrosc. 2006;236: 151-157.

[78] Perrin A., Valentin A., Daumont L., New analysis of the 2v4, v4+v6, 2v6, v3+v4, v3+v6, v1, v5, v2+v4, $2 n 3, v 2+v 6$ and $v 2+v 3$ bands of formaldehyde H2 12C16O: Line positions and intensities in the 3.5 $\mathrm{mm}$ spectral region Journal of molecular structure 2006;780-781: 28-44.

[79] Flaud J., Lafferty W., Sams R., Sharpe S., High resolution spectroscopy of H2 $12 \mathrm{C} 160$ in the 1.9 to $2.56 \mu \mathrm{m}$ spectral range Molec. Phys. 2006;104: 1891.

[80] Bouwens R.J., Hammerschmidt J.A., Grzeskowiak M.M., Stegink T.A., Yorba P.M., Polik W.F., Pure vibrational spectroscopy of S 0 formaldehyde by dispersed fluorescence J. Chem. Phys. 1996;104: 460.

[81] Perrin A., Keller F., Flaud J.-M., New analysis of the v2, v3, v4, and v6 bands of formaldehyde H2 $12 \mathrm{C} 160$ line positions and intensities in the 5-10 Im spectral region J. Molec. Spectrosc. 2003;221: $192-198$.

[82] Perrin A., Flaud J.-M., Predoi-Cross A., Winnewisser M., Winnewisser B.P., Mellau G., Lock M., New High-Resolution Analysis of the v3 , v4 , and v6 Bands of D2CO Measured by Fourier Transform 
Spectroscopy J. Molec. Spectrosc. 1998;187: 61-69.

[83] Rey M., Group-theoretical formulation of an Eckart-frame kinetic energy operator in curvilinear coordinates for polyatomic molecules J. Chem. Phys. 2019;151: 024101.

[84] Rey M., Nikitin A.V., Tyuterev V.G., Complete nuclear motion Hamiltonian in the irreducible normal mode tensor operator formalism for the methane molecule J. Chem. Phys 2012;136, no. 24: 244106.

[85] Rey M., Nikitin A.V., Tyuterev VI.G., First principles intensity calculations of the methane rovibrational spectra in the infrared up to $9300 \mathrm{~cm}-1$. Phys. Chem.Chem. Phys. 2013;15, no. 25: 10049-10061.

[86] Rey M., Nikitin A.V., Tyuterev VI.G., Convergence of normal mode variational calculations of methane spectra: Theoretical line list in the icosad range computed from potential energy and dipole moment surfaces J. Quant. Spectrosc. Radiat. Transfer. 2015;164: 207-220.

[87] Lohilahti J. , Alanko S., The v2 Band of Formaldehyde-d2 J. Molec. Spectrosc. 2001;205: 248-251.

[88] Lohilahti J., Ulenikov O.N., Bekhtereva E.S., Alanko S., Anttila R., High resolution infrared study of D2CO in the region of $1780-2400 \mathrm{~cm}-1$ : assignment and preliminary analysis J.Molec. Structure 2006;780-781: 182-205.

[89] Tyuterev VI.G., Tashkun S.A., Rey M., Kochanov R.V., Nikitin A.V., Delahaye T., Accurate spectroscopic models for methane polyads derived from a potential energy surface using highorder contact transformations. J.Phys.Chem. A 2013;117: 13779-13805.

[90] Nikitin A.V., Thomas X., Daumont L., Rey M., Sung K., Toon G.C., Smith M.A.H., Mantz ProtasevichA.E., A.W., Tashkun S.A., Tyuterev VI.G., Assignment and modelling of $12 \mathrm{CH} 4$ spectra in the 5550-5695, 5718-5725 and 5792-5814 cm-1 regions J. Quant. Spectrosc. Radiat. Transfer 2018;219: 323-332.

[91] Nikitin A.V., Protasevich A.E., Rey M., Tyuterev V.G., Highly excited vibrational levels of methane up to $10300 \mathrm{~cm}-1$ : Comparative study of variational methods J. Chem. Phys. 2018;149: 124305. 